\title{
High-throughput functionalization of the Toxoplasma kinome uncovers a novel regulator of invasion and egress
}

\author{
Tyler A. Smith ${ }^{1,2}$, Gabriella S. Lopez-Perez², Emily Shortt"1 , Sebastian Lourido ${ }^{1,2, *}$ \\ ${ }^{1}$ Whitehead Institute for Biomedical Research, Cambridge, MA 02142, USA \\ ${ }^{2}$ Department of Biology, Massachusetts Institute of Technology, Cambridge, MA 02139, USA \\ *Correspondence: lourido@wi.mit.edu
}

\begin{abstract}
Protein kinases regulate fundamental aspects of cell biology in all eukaryotes, making them attractive chemotherapeutic targets in Apicomplexan parasites such as the causative agents of malaria (Plasmodium spp.) and toxoplasmosis (Toxoplasma gondii). However, the precise roles of individual parasite kinases cannot be inferred simply from sequence identity, due to rewiring of signaling pathways and the shifting repertoire of kinases across species. To systematically examine the parasite kinome, we developed a high-throughput (HiT) CRISPR-mediated tagging strategy to endogenously label all predicted cytosolic protein kinases with a synthetic sequence encoding the minimal auxin-inducible degron (mAID) linked to a fluorophore and epitope tag. The system enables the assembly of thousands of tagging vectors from synthetic sequences in a single reaction and the pooled generation of mutants to examine kinase localization and function. We examined the phenotypes associated with kinase knock-down in 1,160 arrayed clones by replica-plating in the presence or absence of auxin and found broad defects across the lytic cycle for 109 clonal isolates, assigning localizations to 39 proteins, and associating 15 kinases within 6 distinct morphological phenotypes. The relative fitness of tagged alleles was also examined by tracking the relative abundance of individual guide RNAs as parasite populations progressed through the lytic cycle, in the presence or absence of auxin. Pooled screening had a high predictive value and differentiated between delayed and acute death. Demonstrating the value of this resource, we identified a novel kinase associated with delayed death as a novel regulator of invasion and egress. We call the previously unstudied kinase Store Potentiating/Activating Regulatory Kinase (SPARK), based on its impact on intracellular $\mathrm{Ca}^{2+}$ stores at key moments during the lytic cycle. Despite having a similar kinase domain to the mammalian PDK1, SPARK lacks the canonical lipid-binding domain and we find no indication SPARK positively regulates other AGC kinases, suggesting a rewiring of signaling pathways to accommodate parasite adaptations. The HiT vector screening system extends the applications of genome-wide screens into complex cellular phenotypes, providing a scalable and versatile platform for the dissection of apicomplexan cell biology.
\end{abstract}

\section{INTRODUCTION}

Apicomplexans are widespread parasites that include the causative agents of toxoplasmosis (Toxoplasma gondii), cryptosporidiosis (Cryptosporidium spp.), and malaria (Plasmodium spp.). Despite their global health burden, many aspects of apicomplexan cell biology remain poorly understood. Kinases can provide important insight into parasite biology as key regulators of cellular processes that are consequently attractive drug targets ${ }^{1-3}$. However, in silico inference of kinase function is challenging since evolutionary divergence and parasitespecific adaptations drive the rewiring of signaling pathways away from their established roles 
in other systems. Even broadly conserved kinase families like the cyclin-dependent kinases (CDKs) have acquired dramatic lineage-specific adaptations ${ }^{4}$. The apicomplexan kinome also contains phylum-specific ROPK, FIKK, and WNG families of secreted kinases that evolved to interfere with the signaling of host cells ${ }^{5-11}$. While many studies have explored the function of individual kinases in isolation, systematic approaches are needed to achieve a global view of the relative contribution of individual enzymes to parasite biology.

Recent high-throughput gene knockout screens in both T. gondii and Plasmodium spp. have provided insight into the fitness costs associated with loss of each gene for parasite growth ${ }^{12-14}$. The incorporation of molecular barcodes has enabled these approaches to profile thousands of genes in a single experiment. Efforts directed specifically at the Plasmodium berghei kinome identified requirements of parasite progression through the sexual cycle ${ }^{15}$. However, detailed high-throughput functional characterization and identification of complex cellular phenotypes beyond general fitness costs remains a critical challenge. Moreover, the lack of temporal control over the implemented gene disruption methods prevents precise timing of essential gene functions. These screens also lack information on protein localization, which can provide insight into a kinase's molecular function.

Several genetic systems have been developed for the precise regulation of $T$. gondii gene expression, enabling the functional analysis of individual genes. Transcriptional regulation systems provide inducible transcriptional control over target genes using heterologous promoters, but regulation may require several replication cycles ${ }^{16-18}$. Conditional genome rearrangements are also possible using a rapamycin-dimerizable Cre recombinase (DiCre) system, to delete entire loci or modify mRNAs for post-transcriptional regulation. DiCre systems can largely retain endogenous regulation, but recombination is irreversible and, like transcriptional regulation, its effects may be delayed for several replication cycles ${ }^{19-21}$. By contrast, the auxin-inducible degron (AID) system confers post-translational regulation, often achieving complete knockdown of the target protein within one hour ${ }^{22-24}$. The AID system is also reversible, maintains the target gene's native promoter, and can be easily paired with a fluorescent protein tag. Deploying the AID system at scale could provide both subcellular localization and temporal resolution for the examination of phenotypes associated with target gene loss.

High rates of non-homologous end joining (NHEJ) typically preclude efficient homologous recombination (HR) in T. gondii. In fungi, mutants deficient in NHEJ have been used for genome engineering, taking advantage of their improved rates of HR ${ }^{25-29}$. Deletion of $T$. gondii KU80, required for NHEJ, likewise greatly increased the efficiency of modifying endogenous loci 30,31. The high HR efficiency of yeast has enabled large-scale gene tagging efforts to generate arrayed strain collections for protein localization by live-cell fluorescence microscopy or interaction networks by immunoprecipitation mass spectrometry ${ }^{32-34}$. Recently, similar efforts have been undertaken in mammalian systems ${ }^{35,36}$ providing critical insight into the function of genes beyond their general fitness cost. In parasites, arrayed protein localization surveys have been carried out in the kinetoplastid Trypansoma brucei; however, no such approach has been developed for an apicomplexan parasite ${ }^{37}$. Stable NHEJ-deficient strains, high rates of transfection, and the adaptation of CRISPR/Cas9-based genome engineering ${ }^{38}$ make T. gondii ideal for the development of high-throughput tagging methods.

We present a high-throughput tagging $(\mathrm{HiT})$ strategy which, paired with AID conditional knockdown in T. gondii, is amenable to both arrayed and pooled screening. We profiled the kinome of $T$. gondii assessing subcellular localization, and defects in cell division and the 
Iytic cycle. Our system provides spatiotemporal resolution that led to the discovery of novel regulators of various lytic cycle checkpoints, including a previously unstudied kinase critical for invasion and egress from host cells. Our system will be a powerful tool for systematically dissecting apicomplexan cell biology.

\section{RESULTS}

\section{Development of high-throughput tagging vectors for T. gondii}

While pooled knockout screening can identify general fitness phenotypes ${ }^{12}$, it fails to capture protein localization and expression levels, and the precise timing of phenotype development. To that end, we developed a high-throughput tagging (HiT) strategy that uses CRISPRdirected homologous-recombination to efficiently and site-specifically integrate exogenous sequences (payloads), such as epitope tags or regulatable elements (Fig. 1A). This strategy is scalable thanks to rapid cloning from entirely synthetic sequences, a modular design to introduce alternative payloads, and the flexibility to construct vectors against multiple sites in a pooled format. HiT vectors encode a gRNA targeting the $3^{\prime}$ end of a gene as well as two $40 \mathrm{bp}$ homology regions specific to the target site. Proper HR-mediated repair of the double-stranded break integrates the synthetic sequence into the genomic locus alongside a selectable marker, eliminating the gRNA target site. Integration of the gRNA sequence, along with the rest of the construct, introduces a molecular barcode through which each mutant can be identified. Because all targeting sequences are contained within a single vector, the strategy is compatible with both pooled and arrayed screening.

To examine the efficiency of the HiT strategy, we designed HiT vectors to tag genes with the $\mathrm{mNeonGreen}(\mathrm{mNG})$ fluorophore fused to a minimal auxin-inducible degron (mAID). In T. gondii strains containing the heterologous F-box protein TIR1, addition of the auxin indole3-acetic acid (IAA) leads to ubiquitination and proteasomal degradation of the mAID-tagged protein $22,23,39,40$. This payload achieves both localization and regulated degradation of the gene product. As test cases, we targeted the kinases CDPK1 and CDPK3, as examples of an essential and a dispensable gene, respectively. Both kinases tolerate C-terminal tags ${ }^{41-43}$. We also constructed scarless clones in which the tag was integrated into either the CDPK 1 or the CDPK3 locus in the absence of a marker or other exogenous sequences; these control strains provide a reference to calibrate the expression of each gene from strains constructed with our HiT vectors. TIR1 parasites were transfected with HiT vectors targeting each gene together with a Cas9-expression plasmid. After selection, a majority of parasites in each population was mNeonGreen positive as assayed by flow cytometry (Fig. 1B). However, HiT-tagged populations were only half as fluorescent as their corresponding scarless counterparts. We hypothesized that the decreased expression may be due to the heterologous UTR selected for the vector. Replacement of the SAG13' UTR $\left(3^{\prime}{ }_{\text {SAG1 }}\right)$ with the CDPK3 $3^{\prime}$ UTR $\left(3^{\prime}{ }_{\text {CDPK3 }}\right)$ recovered the expression of the HiT-tagged alleles to near wild-type levels for both CDPK1 and CDPK3 (Fig. 1B; bottom row). The UTR-dependent changes in expression were also apparent by livecell microscopy (Fig. 1C), which additionally confirmed the expected localization of the two kinases ${ }^{41,43}$. Confirming the function of the tag, treatment with IAA for 24 hours resulted in complete depletion of the protein tagged in each population (Fig. S1).

To further characterize the source of heterogeneity within the selected populations, we isolated $10 \mathrm{mNeonGreen-positive} \mathrm{and} 10 \mathrm{mNeonGreen-negative} \mathrm{clones} \mathrm{from} \mathrm{each} \mathrm{of} \mathrm{the} \mathrm{tagged} \mathrm{CDPK1}$ 
bioRxiv preprint doi: https://doi.org/10.1101/2021.09.23.461611; this version posted September 24, 2021. The copyright holder for this preprint (which was not certified by peer review) is the author/funder, who has granted bioRxiv a license to display the preprint in perpetuity. It is made available under aCC-BY-NC-ND 4.0 International license.

A

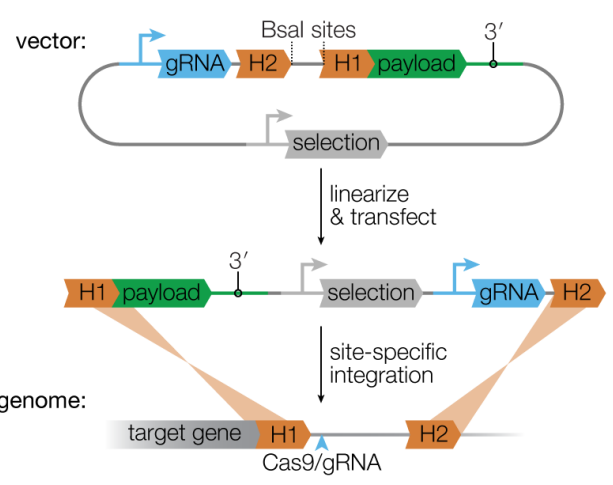

B

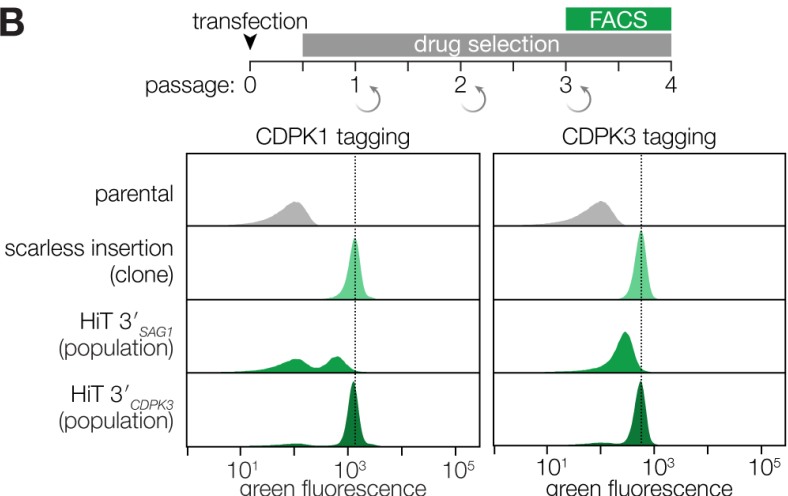

C

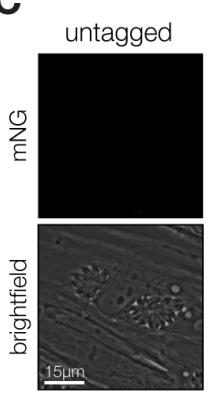

CDPK1 tagged

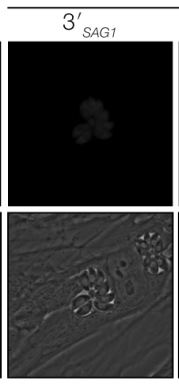

CDPK3 tagged

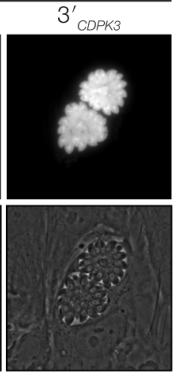

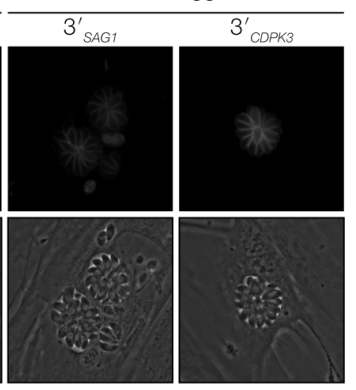

D

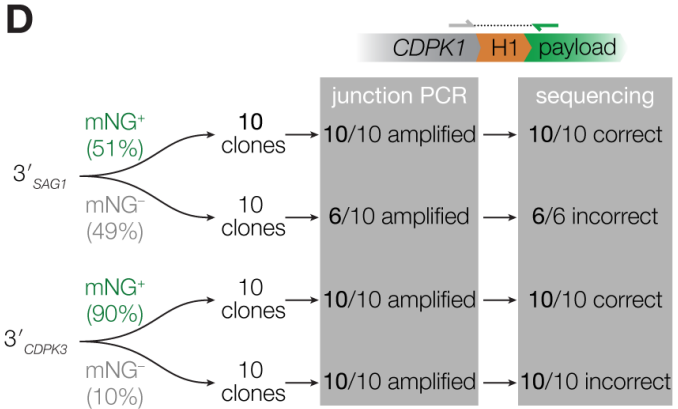

$\mathbf{E}$

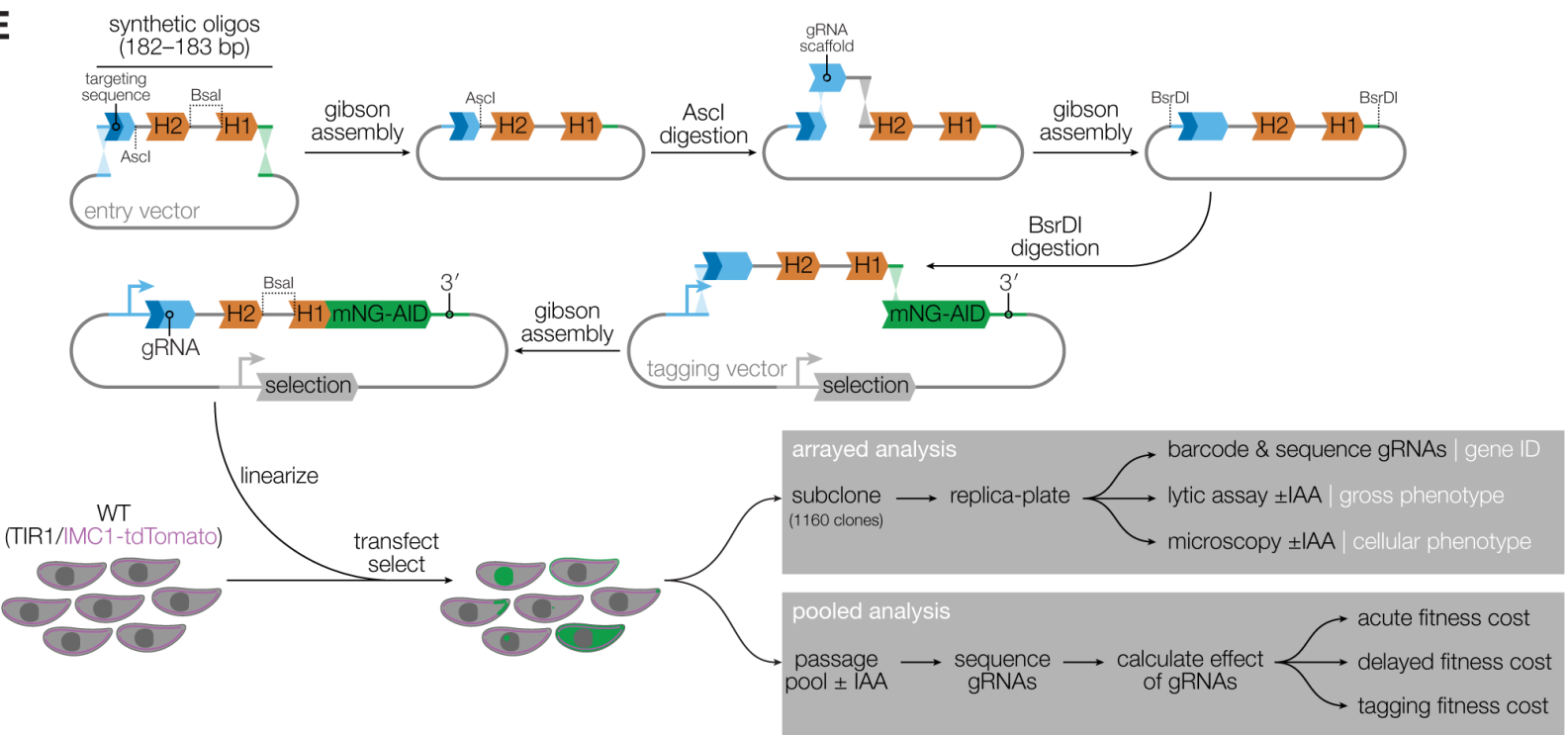

Figure 1. Development of high-efficiency tagging (HiT) constructs for protein-centered screening approaches. (A) Schematic of the high-throughput tagging $(\mathrm{HiT})$ vector. The Bsal-linearized vector is cotransfected with a Cas9-expression plasmid into parasites to mediate homologous recombination of the construct into the target locus. $\mathrm{H} 1$ and $\mathrm{H} 2$ indicate $40-$ $\mathrm{bp}$ regions of homology to the target locus. Pyrimethamine selection was used to isolate successful integration events. A heterologous 3' UTR is included in the vector following the tagging payload. (B) Efficiency of HiT vector tagging. Parental TIR1 parasites were transfected with HiT $3^{\prime}{ }_{\text {SAG1 }}$ or HiT $3^{\prime}{ }_{\text {CDPK3 }}$ vectors targeting CDPK 1 or CDPK3 with a tagging payload encoding mNeonGreen fused to the minimal auxin-induced degron (mAID). Following selection, the populations were analyzed by flow cytometry for mNeonGreen fluorescence and compared to clonal strains carrying the same tag with no exogenous sequences (scarless insertion). Dotted line centered on the mode for the fluorescence of the scarless insertion. (C) Fluorescence microscopy of the tagged populations displaying the correct localization of each kinase and expression levels consistent with the flow cytometry results (B). Brightness of all mNeonGreen (mNG) images was equally adjusted. (D) The 5' integration junctions of $10 \mathrm{mNG}$ positive and $10 \mathrm{mNG}$ negative clones from the CDPK1 HiT $3^{\prime}{ }_{\text {SAG } 1}$ and HiT $3^{\prime}{ }_{\mathrm{CDPK} 3}$ populations were amplified and sequenced. Junctions were categorized according to whether they could be amplified by PCR (junction PCR) and exhibited the correct sequence at the recombination site (sequencing). (E) Schematic of HiT $3^{\prime}{ }_{\text {CDPKз }}$ vector library construction and screening strategy. Following construction and linearization of the library, the vector was co-transfected with a Cas9 expression plasmid into parasites expressing the TIR1 ligase and a fluorescent peripheral marker (TIR1/IMC1-tdTomato). Following selection, the population was analyzed by both pooled and arrayed screening. 
populations, and sequenced the gene-tag junction. In all cases, the mNeonGreen-positive clones from either HiT construct had the tag successfully integrated in frame. By contrast, the junction could only be amplified from 6 of the 10 negative clones originating from the population that used the $3^{\prime}{ }_{S A G 1}$ HiT vector; in all such cases, the vector integrated into the correct locus with a frameshift mutation that precluded tag expression. Analogously, all $10 \mathrm{mNeonGreen-negative}$ clones from the population that used the $3^{\prime}{ }_{C D P K 3}$ construct had integrations marked by frameshift mutations (Fig. 1D). These results indicate that nearly all of the selected parasites integrated the HiT vectors in the correct locus, with a majority of parasites in the selected population harboring the tag perfectly in frame- $51 \%$ of those selected with the $3^{\prime}{ }_{\text {SAG } 1}$ construct, and $90 \%$ of those selected with the $3^{\prime}{ }_{\mathrm{CDPK} 3}$ construct-rates compatible with high-throughput screening.

\section{Generating an array of conditional mutants}

Protein kinases exhibit a broad range of localizations within Toxoplasma to control key life cycle events such as invasion, replication, and egress ${ }^{6}$. Using the HiT vector platform we chose to profile the parasite kinome. We designed constructs against 147 protein kinases and eight control genes. We included the protein kinase A regulatory subunit (PKAr), Doc2.1, a putative calmodulin, and PI-PLC based on their known or proposed roles in the lytic cycle during entry and exit from the host cell ${ }^{44-47}$. CAM1, CAM2, and CAM3 were also included based on previous literature that successfully tagged these genes with the AID system ${ }^{48}$. Finally, we included the hypothetical protein TGGT1_222305 as a non-kinase dispensable control ${ }^{12}$. We excluded predicted ROP kinases and kinases with predicted signal peptides, based on the prediction that they are likely inaccessible to the cytosolic TIR1, and therefore of limited potential within current screen design. Previous genome-wide screens also indicated that ROP kinases are largely dispensable in cell culture ${ }^{12}$.

For each gene we designed homology regions upstream and downstream from the doublestranded break, which were paired with one of three different gRNAs per gene, resulting in 465 unique constructs. To increase the possible number of gRNAs per gene, we selected gRNAs with either NGG or NAG protospacer adjacent motifs (PAMs). Homology region 1 $(\mathrm{H} 1)$, which leads into the HiT vector tag, was defined as the $40 \mathrm{bp}$ upstream of the stop codon. Because the efficiency of homologous recombination decreases with the distance of the homology regions from the double-stranded break, we only selected gRNAs that cut within a $50 \mathrm{bp}$ window downstream of the stop codon ${ }^{49-52}$. Homology region $2(\mathrm{H} 2)$ was designed to be $40 \mathrm{bp}$ in length and starts $6 \mathrm{bp}$ downstream of the cutsite. This removes the gRNA cutsite after vector integration, preventing futile cycles of cutting. Potential gRNAs were scored and ranked by on-target and off-target cutting frequency using the Rule Set 2 and cutting frequency determination algorithms ${ }^{53}$ and by their distance from the gene's stop codon. These three metrics were used to create an aggregate rank, from which the three highest-ranking gRNAs were chosen for each gene.

Since the screens were performed in parallel to the analysis of UTR function, we generated libraries using the two different $3^{\prime}$ UTRs. The first library was created with the $3^{\prime}{ }_{\text {SAG } 1}$ HiT vector, and the second library was created with the $3^{\prime}{ }_{\text {CDPK } 3} H$ HiT vector. Short oligos encoding the gRNA and matched homology regions were synthesized by massively parallel oligonucleotide synthesis and cloned into an entry vector. The library in the entry vector was linearized with Ascl to introduce the gRNA scaffold, which exceeded the size constraints for oligonucleotide synthesis. The complete gRNA and homology regions were then cloned as a unit into the 
HiT vector to generate the final libraries. For the $3^{\prime}{ }_{S A G 1}$ library, the assembled units were PCR amplified from the entry vector; however, we observed recombination leading to chimeric vectors containing gRNAs and homology regions targeting different genes. To reduce such PCR artifacts, the $3^{\prime}{ }_{\mathrm{CDPK} 3}$ library was cloned using a modified entry vector from which the assembled units could be excised with a restriction enzyme (BsrDI) and directly cloned into the HiT vector (Fig. 1E). The final strategy helps maintain library diversity while minimizing errors produced by repeated rounds of PCR amplification, thereby generating thousands of tagging vectors in a single reaction.

We carried out both arrayed and pooled screens using the constructed libraries. While pooled screening provides an easily scalable platform to compare the fitness of mutants under various conditions, arrayed screening enables precise phenotype determination through replica plating and fluorescence microscopy. To generate the clonal array, the $3^{\prime}{ }_{\text {CDPK } 3}$ HiT vector library was transfected into parasites expressing TIR1, for conditional degradation of the AID-tagged proteins 22-24,48. The parasites additionally expressed a red fluorescent inner membrane complex marker (IMC1-tdTomato) to visualize the parasite ultrastructure across the replicative cycle ${ }^{54}$. Following transfection and selection of the pooled $3^{\prime}{ }_{\mathrm{CDPK} 3}$ HiT vector library, the population was subcloned by limiting dilution. 1160 clonal strains were arrayed and passaged in 96-well plates (Fig. 1E). As controls, each plate was seeded with the parental TIR1 strain and a strain in which CDPK1 was endogenously tagged with the AID payload.

Sanger sequencing is cost prohibitive for the deconvolution of large numbers of clones. We therefore employed dual-indexing PCR to sequence the gRNAs from all 1160 clonal lines in a single next-generation sequencing experiment. Using barcoded forward and reverse primers, the integrated gRNAs of each well were amplified with a specific dual-index combination, allowing us to assign gRNA reads to each well of the array. A well was designated as gRNAcontaining if a single gRNA had more than 100 reads. Wells containing multiple integrations or mixed populations were defined as those in which a secondary gRNA contained more than $10 \%$ the number of reads assigned to the most abundant gRNA. We were able to assign gRNA identities to $79 \%$ (917) of all wells, with the remaining $21 \%$ failing to amplify (Fig. 2A). $87 \%$ (796) of the amplified wells contained a single gRNA, representing 49\% (228) of gRNAs in the original library and 82\% (127) of the targeted genes (Fig. 2B). 13\% (121) of amplified wells contained two or more gRNAs. To prevent confounding effects from double-tagged strains, only singly tagged clones were used for phenotype and localization assignment.

\section{Arrayed screening to determine protein localizations and phenotypes}

We employed high-content imaging to determine the localization of tagged proteins, visualize their depletion following IAA treatment, and observe the cellular consequences of their knockdown. The arrayed clones were replica-plated into 96-well glass-bottom plates containing human foreskin fibroblast (HFF) monolayers, treated with IAA or vehicle 3 hours post-infection, and imaged the following day. A clear mNeonGreen signal was observed in 29\% (232) of singly tagged clones, allowing us to unambiguously determine the localization of 39 proteins, representatives of which were imaged by confocal microscopy (Fig. 2C, Fig. S2, Table S1). Based on the stereotypical polarized morphology of the parasites and the IMC marker, proteins could be assigned to a wide variety of subcellular compartments including nucleus (11), cytosol (9), apical end (5), cellular periphery (3), perinuclear space (2), basal end (1), daughter-cell IMC (1), parasitophorous vacuole (1), and other punctate structures (6). 36 of the 
bioRxiv preprint doi: https://doi.org/10.1101/2021.09.23.461611; this version posted September 24, 2021. The copyright holder for this preprint (which was not certified by peer review) is the author/funder, who has granted bioRxiv a license to display the preprint in perpetuity. It is made available under aCC-BY-NC-ND 4.0 International license.
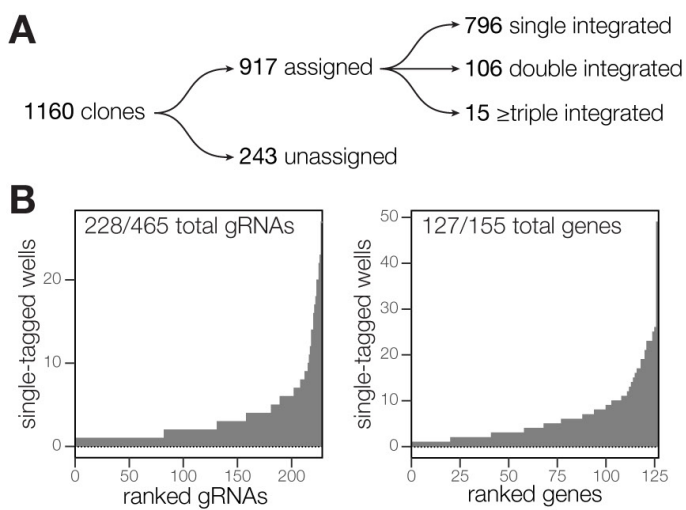

D
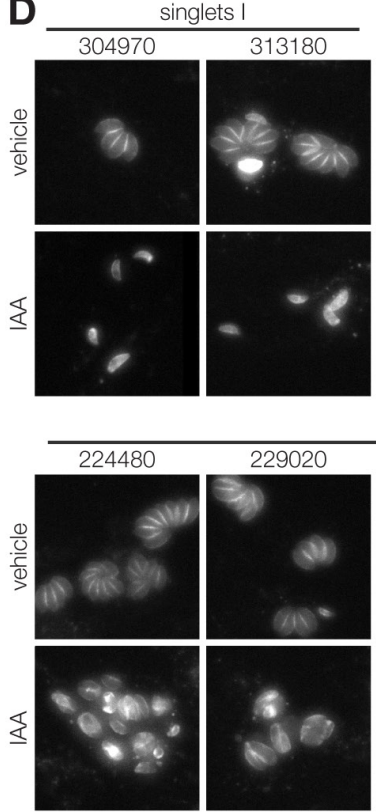

cell division II
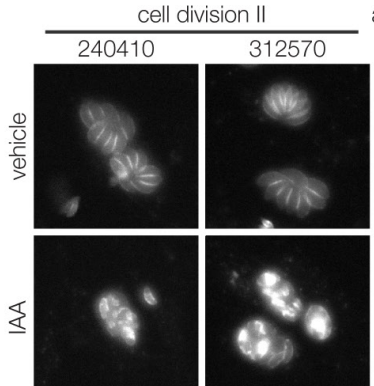
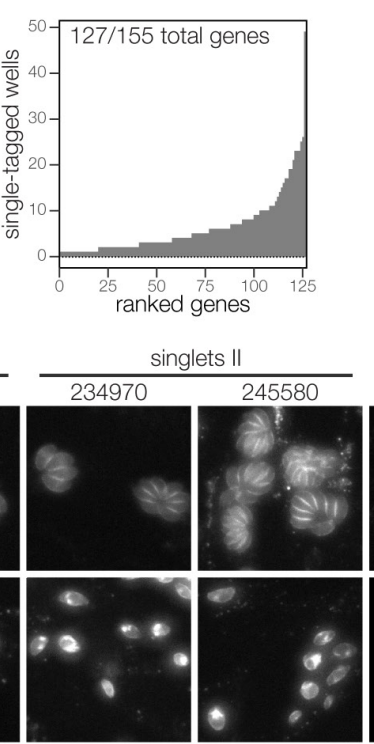

cell division
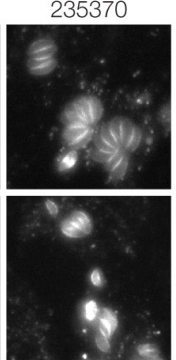

accumulated IMC
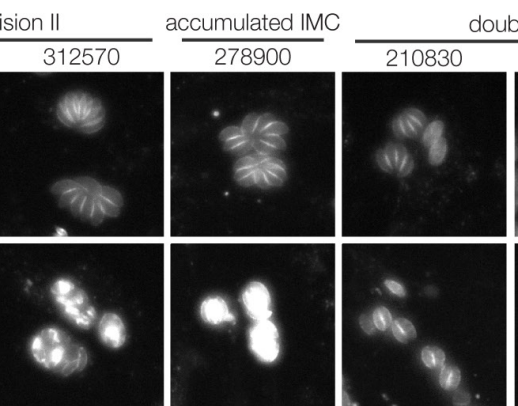
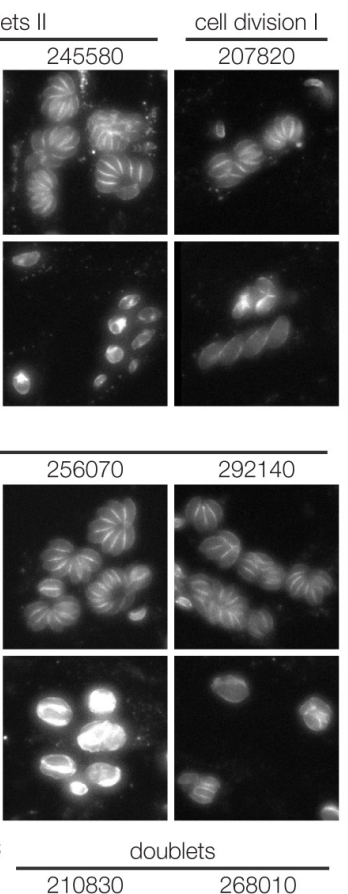

$\mathrm{E}$
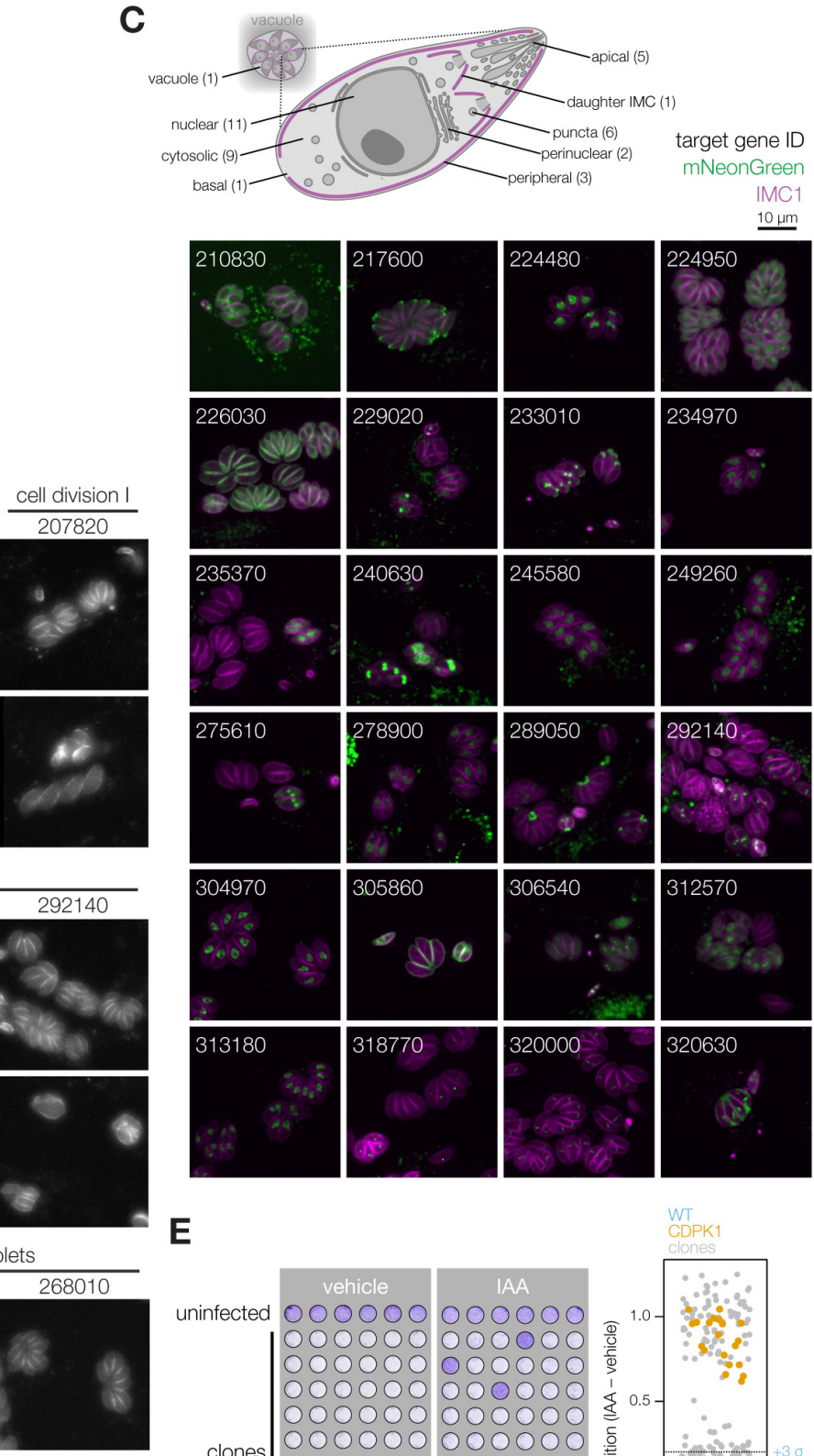

Figure 2. Deconvolution of protein phenotypes and localizations through high-content imaging of arrayed HiT clones. (A)

Results from dual-indexed sequencing of the arrayed clones. A minimum of 100 reads were required to assign a given gRNA to a particular clone. Cases where a second gRNA reached $>10 \%$ the abundance of the first gRNA were classified as containing multiple integrations. (B) Histogram showing the distribution of gRNAs and genes contained among single-integrated wells within the array. Only gRNAs and genes with an instance of at least 1 are plotted. (C) Distribution of localizations contained in the array; number of proteins found in each subcompartment indicated in parentheses. Localizations were assigned to a gene if half or more of single-integrated wells for that gene displayed consistent localizations. Displayed are representative confocal images of a sample of clones. mNeonGreen (green); IMC1-tdTomato (magenta). Genes are numbered based on the unique identifier from ToxoDB (e.g. TGGT1_210830, labeled 210830). (D) Widefield microscopy of representative clones with identified phenotypes. The IMC1-tdTomato marker is displayed for cultures treated with either vehicle or IAA for $24 \mathrm{~h}$. Phenotypes were binned into 6 categories based on their similarity. (E) The ability of clones to lyse host cell monolayers was assayed by infecting monolayers for $72 \mathrm{~h}$ in the presence or absence of IAA. Intact monolayers were visualized by crystal violet staining. Normalized absorbance measurements comparing vehicle- and IAA-treated wells are graphed for each clone. Each plate contained the parental strain (WT) and an AID-tagged CDPK1 clone (CDPK1) as controls. Mean + S.D. for WT controls are shown. 
39 proteins observed showed complete depletion after 24 hours of IAA treatment. By contrast, TGGT1_322000 exhibited only partial depletion, and TGGT1_320630 and TGGT1_234950 showed no signal reduction under IAA treatment. Since TGGT1_320630 localized to the parasitophorous vacuole and TGGT1_234950 was localized to dense granules by HyperLOPIT ${ }^{55}$, lack of degradation likely resulted from inaccessibility to the TIR1 machinery. Our results illustrate how the HiT screening strategy can be used to determine the localization of parasite genes in a high-throughput manner.

We were able to detect knockdown-induced phenotypes, using IMC1-tdTomato as a marker for parasite structure in the IAA-treated wells to categorize mutants based on their effects on replication and overall morphology (Fig. 2D). Clones bearing gRNAs against TGGT1_304970, TGGT1_313180, TGGT1_234970, or TGGT1_245580 arrested early in the lytic cycle and were mostly observed as single parasites within host cells. Degradation of the latter two additionally caused a discontinuity in the IMC marker (singlets II). Consistent with our results, a previous study categorized TGGT1_304970 as a nuclear cyclin-related kinase (TgCrk1) and demonstrated that its loss caused abnormal assembly of the daughter cell cytoskeleton and failure to progress through the cell cycle ${ }^{56}$. TGGT1_234970 was also previously localized to the nucleus and categorized as the tyrosine kinase-like protein TgTKL2 ${ }^{57}$. Although the function of TgTKL2 has not been determined, its similarity to the human Tousled-like kinase TIk2 suggests it may serve a similar role promoting DNA replication ${ }^{58-60}$, which would be consistent with the early arrest we observed. The two other kinases that caused an early arrest also localize to the nucleus but have not been studied in detail. TGGT1_313180 appears to be an ortholog of PRP4, which in fission yeast regulates pre-mRNA splicing and its inhibition leads to transient arrest during the gap phases of the cell cycle ${ }^{61}$. By contrast, TGGT1_245580 is only conserved among coccidians (e.g. Toxoplasma, Neospora, Hammondia, Eimeria, and Cyclospora) and is predicted to be a large 2,313 residue protein with no annotated features aside from the kinase domain.

We catalogued several other phenotypes involving compromised cell division that resulted in vacuoles of smaller (cell division I) or larger (cell division II) size with aberrant IMC morphologies (Fig. 2D). Within this category, we identified two previously described cyclin-related kinases, TGGT1_256070 (TgCrk4) and TGGT1_229020 (TgCrk5). Consistent with our findings, knockdown of TgCrk4 resulted in major morphological abnormalities and decreased plaque size ${ }^{56}$. Although the function of TgCrk5 has not been previously examined, it was previously localized to the centrocone-consistent with our observations (Fig. 2C) — and shown to interact with the cell cycle regulatory factor ECR $1{ }^{62}$. Strengthening the functional link between the two proteins, knockdown of TgCrk5 in our screen caused the same phenotype observed at the restrictive temperature with a temperature-sensitive allele of ECR $1^{62}$. Other cell division defects were associated with knockdown of the centrosome-associated kinases NIMA-related kinase 1 (TgNek1; TGGT1_292140), MAPK-like protein 1 (TgMAPK-L1; TGGT1_312570), and TgMAPK2 (TGGT1_207820), phenocopying the results of previously characterized temperature-sensitive or auxin-sensitive alleles ${ }^{63-66}$. For TgMAPK-L1, we observe a cell cycle-dependent localization to diffuse puncta, consistent with its reported localization to the pericentrosomal matrix. For TgMAPK2, in addition to the previously reported cytosolic localization ${ }^{66}$, we observe localization to paired puncta in a subset of vacuoles, which may represent cell cycle-regulated recruitment to centrosomes or early daughter cells. This localization sheds additional light into the function of TgMAPK2 and highlights an advantage of utilizing live-cell microscopy. 
Cell cycle defects were also observed for several kinases that have not been previously studied. Knockdown of TGGT1_240410 resulted in a cell division II phenotype comparable to TgMAPK-L1 disruption, despite its limited conservation across closely related coccidians (Neospora and Hammondia). TGGT1_235370 exhibits a similar localization and cell division I phenotype to TgMAPK2. A cell division I phenotype was also observed following TGGT1_224480 knockdown. We show that TGGT1_224480 localizes to the nucleus, similarly to related Cdc-like kinases in mammalian cells that regulate the spliceosome ${ }^{67}$, suggesting a possibly conserved function. In some cases, parasite replication arrested after a single cell cycle. Such doublet phenotypes were observed for knockdown of TGGT1_210830 or TGGT1_268010. Although conservation of TGGT1_268010 appears limited to coccidians, TGGT1_210830 is a putative ortholog of RIO kinase 1, which has been implicated in ribosomal small subunit maturation and cell cycle progression ${ }^{68}$. A distinct phenotype was observed for knockdown of TGGT1_278900, which caused accumulation of the IMC marker with no specific defect in its organization. TGGT1_278900 bears homology to the yeast protein BUD32 and its Plasmodium falciparum orthologue complexes with other components of the EKC/KEOPS complex, suggesting broad conservation of the complex across eukaryotes ${ }^{69}$.

We also screened for aggregate defects in the lytic cycle by examining monolayer clearance after knockdown. Clones were replica-plated onto fibroblast monolayers and treated for 72 hours with vehicle or IAA before quantifying monolayer integrity by measuring the absorbance of crystal violet staining (Fig. 2E). Changes in monolayer clearance were compared to the controls included in every plate: wild-type TIR1 parasites and an AID-tagged CDPK1 mutant that should fail to lyse the monolayer under IAA. Of all 1200 wells, 11\% (129) of the wells scored three standard deviations above the average lysis inhibition score of the 20 WT negative controls, including all 20 CDPK1 positive controls. Based on this metric, these 129 clones represented 21 genes that, when conditionally knocked down, had severe defects clearing the fibroblast monolayers, including every mutant for which phenotypes had been found by high-content imaging except for TgMAPK2, which had displayed an incompletely penetrant phenotype during the time point analyzed. Importantly, clones in which ERK7 or Doc2.1 were tagged showed strong defects in monolayer clearance but displayed no defects by microscopy. This is consistent with their reported roles in invasion and egress but not replication ${ }^{46,70,71 .}$ TGGT1_306540 shared a similar phenotypic profile to ERK7 and Doc2.1 and was previously characterized as an ethanolamine kinase (TgEK) that localized to the parasite cytosol ${ }^{72}$. We similarly observed the cytosolic localization of TgEK and further established its requirement for the completion of the parasite lytic cycle. Taken together, our results highlight the power of arrayed HiT screening to capture both localization and detailed cellular phenotypes that generate new hypotheses about the function of specific kinases.

\section{Pooled screening distinguishes between acute and delayed-death phenotypes}

Arrayed screening successfully identified phenotypes and localizations in clonal populations; however, pooled screening offers greater scalability and a more sensitive comparison of mutant fitness. To perform pooled screens, $3^{\prime}{ }_{\mathrm{SAG} 1}$ or $3^{\prime}{ }_{\mathrm{CDPK} 3} \mathrm{HiT}$ vector libraries were transfected into TIR1 or TIR1/IMC1-tdTomato parasites, respectively. After three passages under pyrimethamine selection, the populations were split and maintained in media supplemented with either vehicle or IAA. At each passage, the parasite populations were sampled to quantify gRNA abundances by next-generation sequencing (Fig. 3A). The abundance of gRNAs in the pre-treatment pool 
bioRxiv preprint doi: https://doi.org/10.1101/2021.09.23.461611; this version posted September 24, 2021. The copyright holder for this preprint (which was not certified by peer review) is the author/funder, who has granted bioRxiv a license to display the preprint in perpetuity. It is made available under aCC-BY-NC-ND 4.0 International license.

correlated strongly with the abundance of gRNAs captured in the array (Fig. S3). We considered three possible influences on the relative abundance of a given gRNA: (i) the efficiency of vector integration, (ii) the tolerance of the locus to tagging, and (iii) the consequence of protein degradation by IAA. As expected from studies on Cas9 targeting efficiency ${ }^{53}$, gRNAs designed to target sequences with NAG PAMs were less abundant than those targeting NGG PAMs in all populations post-selection (Fig. 3B). To regress the effect of the PAM and preserve abundance information related to the effects of tagging on the locus, we normalized the abundance for NAG gRNAs at each time point by the difference in mean abundance between NAG and NGG guides for genes previously considered dispensable ${ }^{12}$ within each sample. Further, we calculated the fold change relative to the second passage, when relative gRNA abundances best reflected the mutant composition in subsequent populations. These transformations allowed us to analyze

A

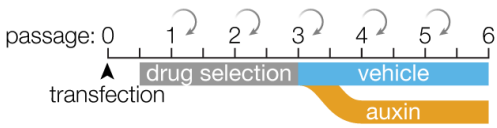

$\mathbf{E}$

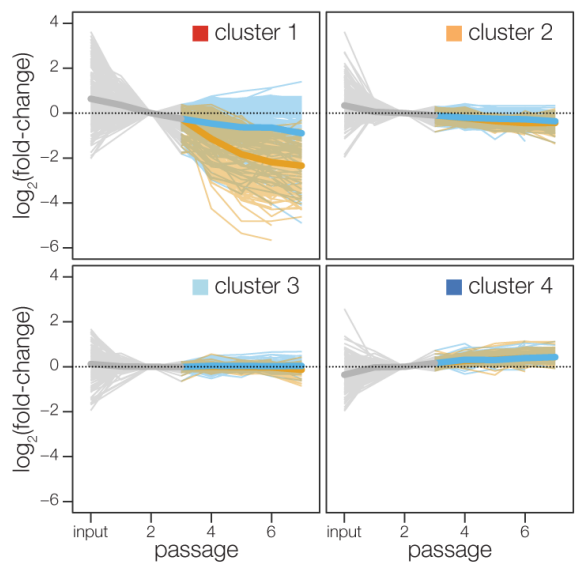

B

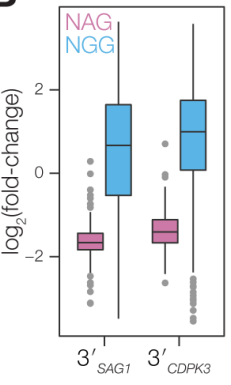

$\mathbf{F}$

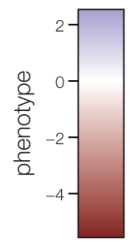

C

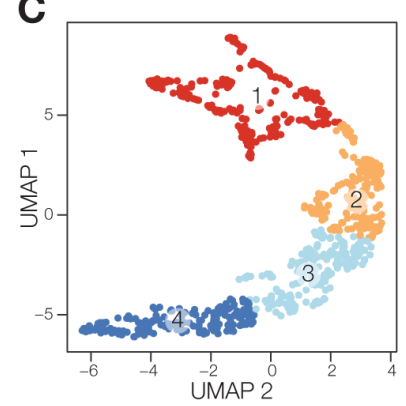

D
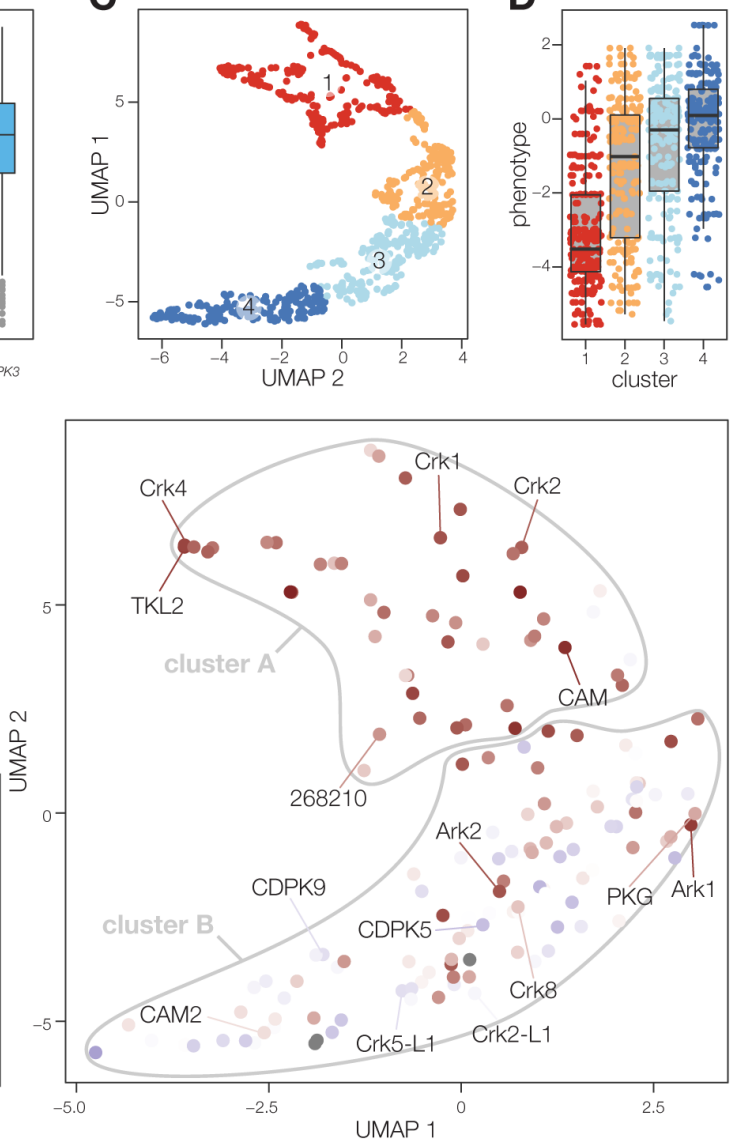

Figure 3. Pooled screening distinguishes between acute and delayed-death phenotypes. (A) Schematic of pooled screening workflow. Transfected populations were selected with pyrimethamine for three passages, after which they were split and cultured in either vehicle- or IAA-containing media. Following each lysis, parasites were split to collect samples for nextgeneration sequencing and continue propagating in fresh host monolayers. (B) Fold-change in gRNA abundance between the vehicle-treated passage 6 and the initial library, plotted by PAM type. Boxplot displays the distribution of each sample by quartiles; outliers highlighted in gray. (C) Relative abundances for each guide were corrected for the effect of the PAM used and fold-changes were normalized to passage 2. UMAP was used to compare guides in each screen based on their pattern of fold changes for vehicle- and IAA-treated samples. Clusters were calculated by k-means. (D) Comparison of phenotype scores from prior gene-disruption screen for each of the clusters in C. Boxplot displays the distribution of phenotypes in each cluster by quartiles. (E) Pattern of fold changes for each guide plotted by cluster. Lines colored by treatment, as in A. Bold lines are the mean for all guides in a given cluster. (F) Gene centroids in UMAP space based on the effects of targeting guides against each gene. Individual genes colored based on the phenotype scores from the prior gene-disruption screen. Genes were assigned to fitness-conferring (cluster A) or dispensable (cluster B) categories based on k-means clustering. (G) Fraction of the maximum fold-change that is explained by the first lytic cycle following IAA addition for all fitness-conferring genes (F). Genes ordered based on the magnitude of effect from acute death to delayed death. 
the results from both screens together, aggregating information from the relative abundance of three gRNAs across both screens for most genes.

We compared the different trajectories for gRNAs across time and IAA treatment. Using UMAP for dimensionality reduction followed by k-means clustering ${ }^{73,74}$, the groupings of gRNAs generated largely agree with previously determined phenotype scores of the target genes (Fig. 3C-D) ${ }^{12}$. Notably, cluster 1 was enriched in gRNAs that differentially dropped out of the population following IAA treatment (Fig. 3E). We therefore expect this cluster contains gRNAs targeting genes whose products are both fitness-conferring and successfully depleted upon IAA treatment.

To integrate the results from multiple gRNAs for the same gene, we calculated the centroid in UMAP space for gRNAs from both screens. Performing unsupervised clustering on the centroids to divide the group into two categories, we observed a cluster of 47 genes (cluster A) that was mainly composed of genes that contribute to parasite fitness (Fig. 3F). Considering that the arrayed screen was performed to capture acute phenotypes, it was encouraging that 18 out of the 22 genes observed to have deficits by microscopy or lytic assay were found in cluster $A$. 13 of the cluster A genes were simply not represented in the array, and the remaining 16 genes that were represented likely display phenotypes that are difficult to appreciate in isolation or during the brief period of kinase depletion examined in the array. One final caveat that may explain discrepancies between the two screens is the possibility that clones characterized in the array resulted from errors in homologous recombination that removed the tag — this may be a common occurrence for genes rendered hypomorphic by the presence of the tag.

Out of the four genes that appeared to have been missed by the pooled screen, for TGGT1_218720 and TGGT1_250680 a single clone was found to have a modest defect in the lytic assay. The two genes likely represent false positives in the arrayed screens, which is supported by their lack of fitness defects in our previous knockout screens ${ }^{12}$. By contrast, the two other genes missed by the pooled screens (TGGT1_278900 and TGGT1_240910) are expected to be fitness-conferring ${ }^{12}$. In the array, Ioss of TGGT1_278900 was associated with the accumulation of the IMC marker (Fig. 2D). Three clones of TGGT1_240910 (Doc2.1) displayed the expected lytic assay phenotype upon knockdown. Doc2.1 was originally included in the library as a control, based on prior studies ${ }^{46}$. For both genes, the discrepancy between screens appears to originate from divergent results between gRNAs. Upon further analysis, each gene had a single gRNA sequence that exhibited reproducible loss in abundance when the population was treated with IAA in each screen; moreover, these were the gRNAs with optimal designs for each gene based on proximity to the coding sequence or use of an NGG PAM.

We next investigated whether the genes predicted to impact fitness in our pooled screens (cluster A) could be further categorized by the timing of gRNA loss. We sorted genes based on the fraction of the maximum effect from IAA treatment that was observed after a single lytic cycle (Fig. 3G). 14 of the 15 genes associated with defects by microscopy (excluding TGGT1_278900, which was missed by the pooled screens) dropped out substantially during the first passage in IAA. Among the genes that dropped out acutely but were not represented in the array, we found TGGT1_270330 (TgCrk7) and TGGT1_215700. TgCrk7 was previously reported to be essential ${ }^{56}$. TGGT1_215700 has not been previously studied but is broadly conserved across eukaryotes and is homologous to phosphatidylinositol 3- and 4-kinases, which are critical for growth and cell proliferation in other organisms ${ }^{75,76}$. Our results suggest that this analysis can 
indeed identify genes whose disruption leads to immediate and catastrophic defects in the parasite.

In contrast to the acute defects associated with some genes, gRNAs against other genes were mostly retained during the first lytic cycle or progressively lost (Fig. 3G). Such delayeddeath phenotypes could be assigned to five genes that have been previously linked to invasion or egress: CDPK1, ERK7, PKAc1, TKL1, and CAM3 24,41,45,47,57,70,71. Consistent with their delayed-death phenotypes, these genes lacked defects during the brief window selected for microscopy of the arrayed clones. Another delayed-death gene, CDPK2a, has not been previously characterized but, like CDPK1, belongs to a kinase family that has been linked to invasion and egress ${ }^{41-43,77-80}$. TgCrk3 also displayed no defects by microscopy or lytic assay, consistent with the observation that knockdown resulted in reduced plaque size but normal morphology ${ }^{56}$. These observations highlight that pooled screens more easily capture subtle defects that accrue over several lytic cycles and may involve the key developmental transitions that accompany egress and invasion.

\section{Analysis of delayed-death genes reveals two kinases that regulate invasion}

We characterized four delayed-death candidates to further understand their roles in the lytic cycle (Fig. 4A). All four kinases were also identified as fitness-conferring in our previous genome-wide knockout screen ${ }^{12}$ and remain largely uncharacterized. The four kinases exhibit different phyletic patterns based on OrthoMCL ${ }^{81}$ : TGGT1_204280 is conserved among several single-celled parasitic phyla, including kinetoplastids (e.g. Leishmania and Trypanosoma spp.), and amoeba (e.g. Naegleria spp.); TGGT1_268210 and TGGT1_239420 are conserved exclusively within the Apicomplexa; and TGGT1_239885 is restricted to coccidians, the clade of apicomplexans to which Toxoplasma belongs. The molecular function of all these kinases remains unclear. However, TGGT1_239420 was previously linked to the development of artemisinin resistance in vitro ${ }^{82}$ and the Plasmodium falciparum ortholog of TGGT1_268210 was recently linked to regulation of protein kinase $A(P K A){ }^{83}$.

For each candidate, we rederived conditional mutants by tagging the endogenous loci with a specific $3^{\prime}{ }_{\mathrm{CDPK} 3} \mathrm{HiT}$ vector. To confirm the pooled screening results, we placed each mutant in competition with TIR1/IMC1-tdTomato parasites under knockdown conditions. A second wildtype strain was used as a control for the assay. All four mutants were outcompeted by the TIR1/IMC1-tdTomato strain, demonstrating the significance of the kinases for parasite fitness

(Fig. 4B). We also assayed the effect of knockdown for each mutant in isolation by plaque assay. Three of the mutants showed clear defects in plaque formation when grown in the presence of IAA; knockdown of TGGT1_239420 or TGGT1_239885 caused severe reductions in plaque size, while knockdown of TGGT1_268210 altogether blocked plaque formation (Fig. 4C). TGGT1_204280 knockdowns exhibited no plaquing defect, suggesting its effect on fitness is either subtle or only revealed in the context of wild-type coinfection. Analogously, parasites lacking TGGT1_239420 or TGGT1_239885 may be readily outcompeted by wild-type parasites due to their reduced fitness.

One possible cause of the delayed-death phenotypes could be a block in invasion. We therefore assayed the invasion efficiency of all four mutants. Both TGGT1_268210 and TGGT1_204280 displayed significant invasion defects upon knockdown (Fig. 4D). Since parasites formed normal plaques upon TGGT1_204280 knockdown, the invasion defect may only represent an overall delay instead of a complete block. By contrast, TGGT1_268210 knockdown caused 
bioRxiv preprint doi: https://doi.org/10.1101/2021.09.23.461611; this version posted September 24, 2021. The copyright holder for this preprint (which was not certified by peer review) is the author/funder, who has granted bioRxiv a license to display the preprint in perpetuity. It is made available under aCC-BY-NC-ND 4.0 International license.
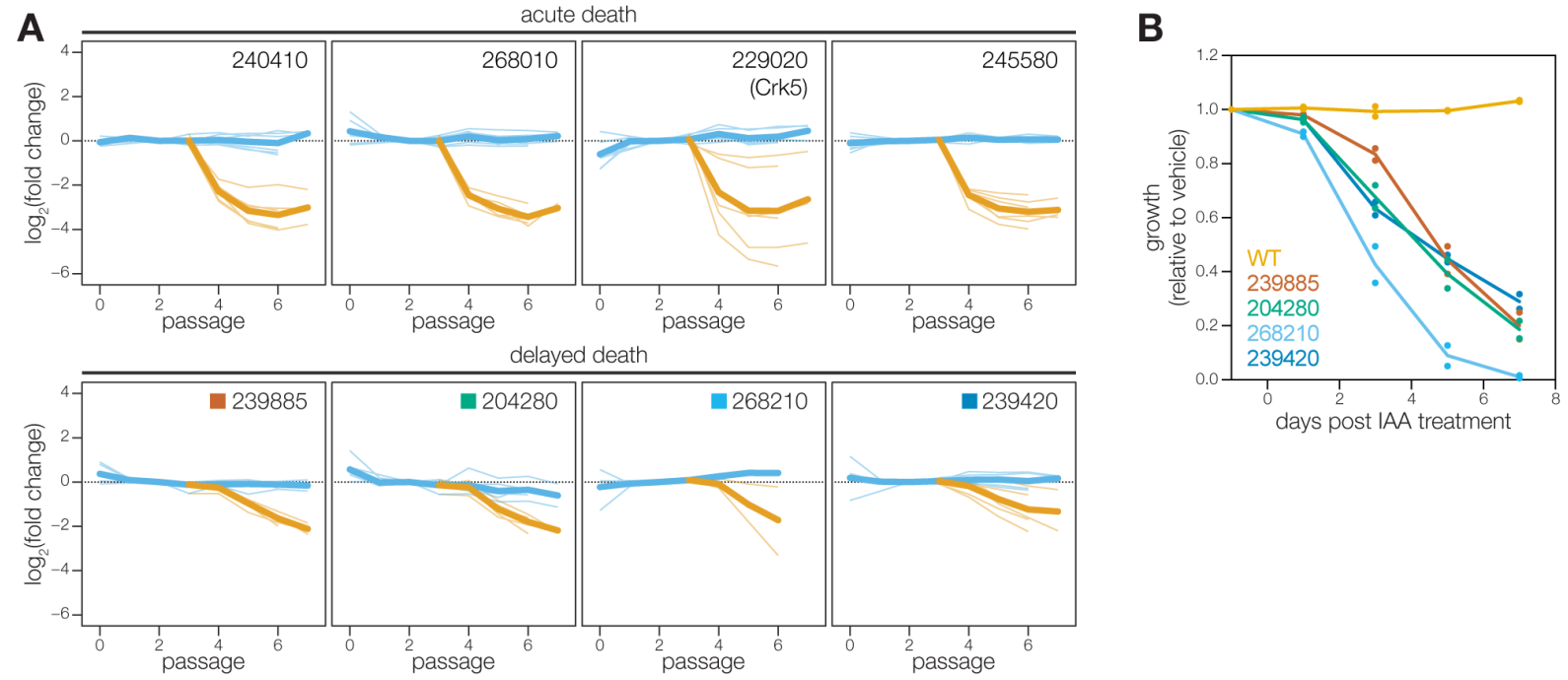

C
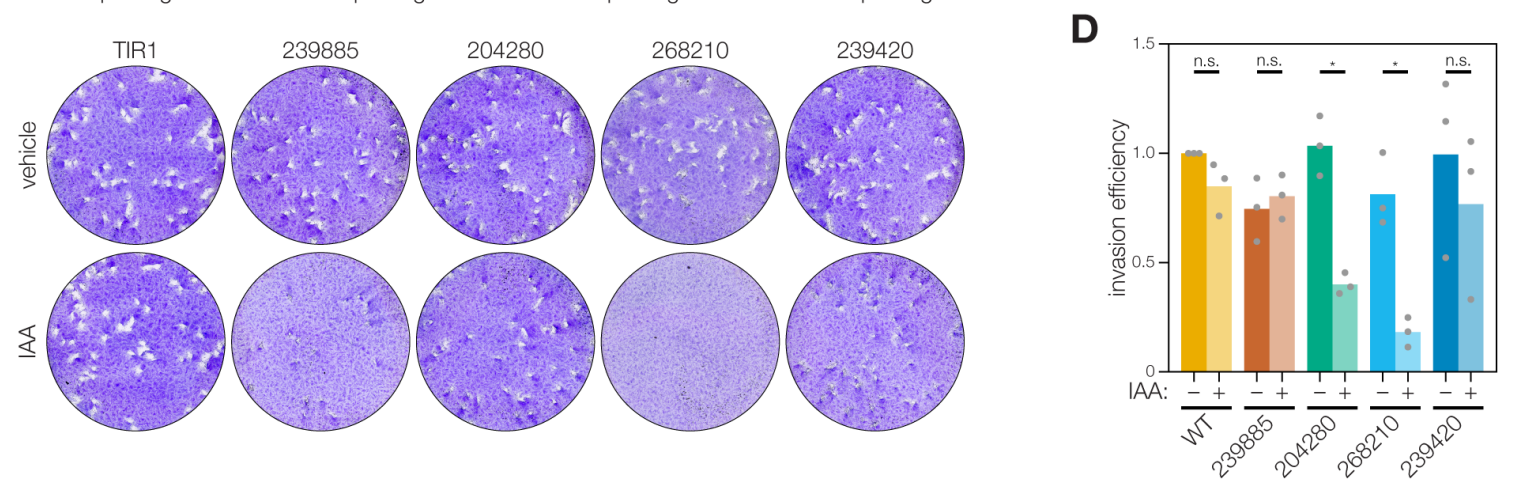

Figure 4. Analysis of delayed-death genes identifies two kinases that impact invasion. (A) Pooled screening traces of selected acute- and delayed-death genes. The fold change of individual guides in the vehicle- (blue) and IAA- (orange) treated samples are displayed. Bold lines are the mean for all guides in a given condition. (B) Competition assays of delayed death candidates, compared the relative growth of AID-tagged strains against a wild-type strain in either vehicle- or IAA-containing media. Following each lysis, the proportion of fluorescent parasites within each competing population was measured by flow cytometry and normalized to the vehicle control. (C) Plaque assays of delayed-death candidates. The ability of candidates to complete the lytic cycle was assayed by plaquing AID-tagged strains for 9 days in either vehicle- or IAA-containing media. Intact monolayers were visualized by crystal violet staining of the monolayers. (D) Invasion assays of delayed death candidates. AID-tagged strains grown in vehicle- or IAA-containing media for $24 \mathrm{~h}$ were incubated on host cells for 10 minutes prior to differential staining of intracellular and extracellular parasites. Parasite numbers were normalized to host cell nuclei for each field. Means graphed for $n=3$ biological replicates; ${ }^{\star} p<0.005$, n.s. $p>0.05$, Welch's one-tailed t-test.

the most severe invasion defect, which coupled with the phenotypes in plaque formation and competition assays suggests the gene plays the most critical role on the lytic cycle among the four candidates with delayed-death phenotypes. These experiments illustrate the value of examining phenotypes with temporal resolution, which helped us identify two novel regulators of invasion based on the pooled HiT screens.

\section{SPARK regulates the release of intracellular $\mathrm{Ca}^{2+}$ stores during egress and invasion}

Based on its critical role during invasion, we examined TGGT1_268210 (here referred to as SPARK) in greater detail. SPARK belongs to the AGC kinase family and orthologs were found throughout the apicomplexan phylum. Although the closest mammalian orthologs of SPARK belong to the 3-phosphoinositide-dependent protein kinase-1 (PDK1) family (Fig. 5A), SPARK lacks the canonical C-terminal phosphoinositide-binding domain found in PDK1, similarly to related kinases in Saccharomyces cerevisiae and nonvascular plants ${ }^{84}$. Instead SPARK and its apicomplexan orthologues possess a strongly conserved N-terminal MKXGFL motif that 
bioRxiv preprint doi: https://doi.org/10.1101/2021.09.23.461611; this version posted September 24, 2021. The copyright holder for this preprint (which was not certified by peer review) is the author/funder, who has granted bioRxiv a license to display the preprint in perpetuity. It is made available under aCC-BY-NC-ND 4.0 International license.

is absent from canonical PDK1s (Fig. 5B). This motif was also conserved in the most closely related orthologues of the free-living alveolates Vitrella brassicaformis and Chromera velia. V. brassicaformis and $C$. velia each also possess a second PDK1-like kinase, both of which lack the N-terminal MKXGFL motif. This suggests that gene duplication may have given rise to the SPARK clade prior to the split of the Apicomplexa from other Alveolata, which was followed by subsequent loss of the more closely related PDK1 kinase in the parasitic clade.

A

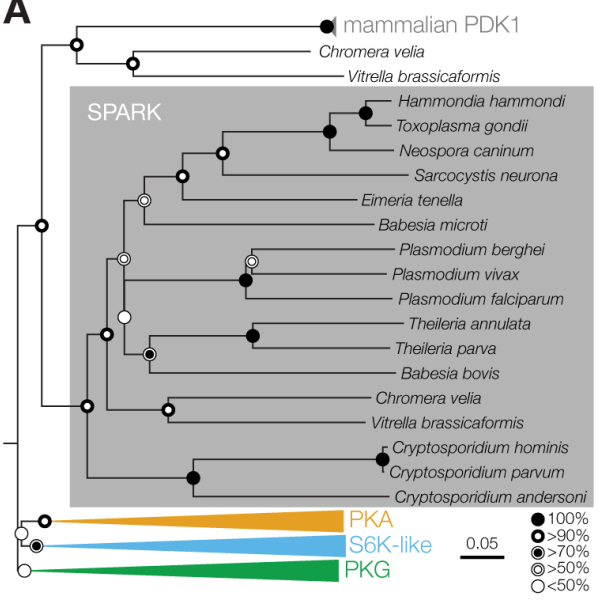

E

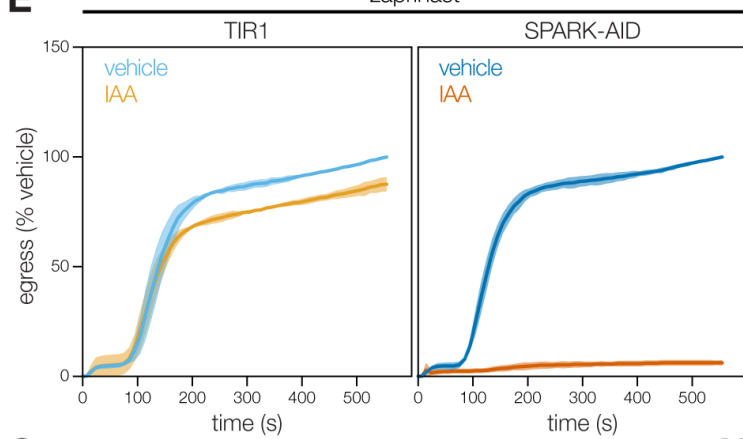

G

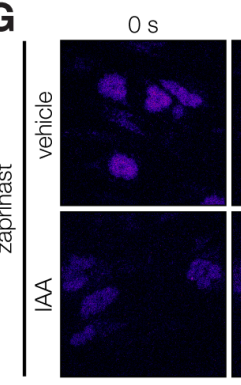

Os
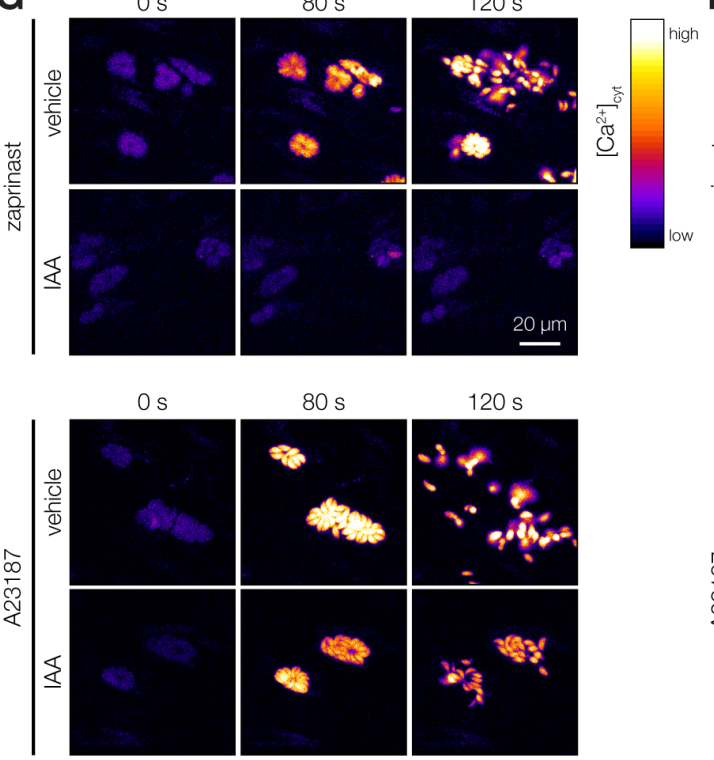

B

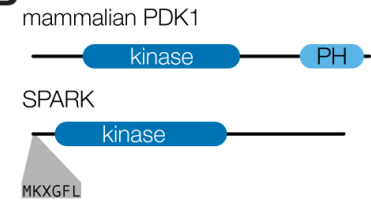

D

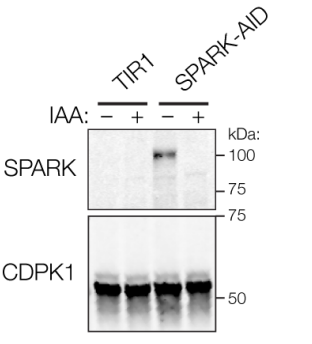

C

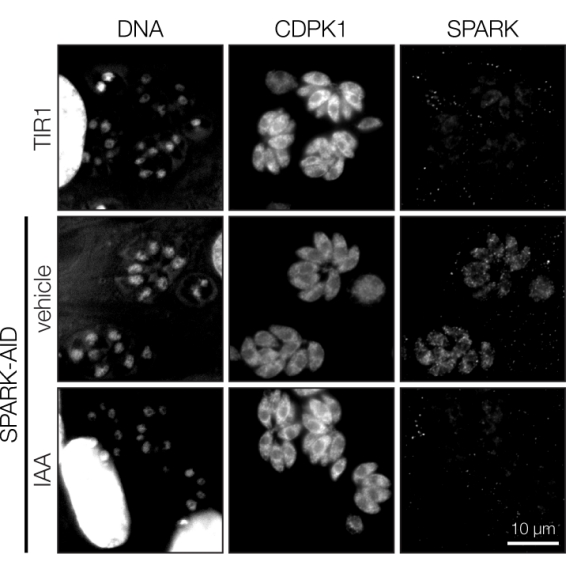

F

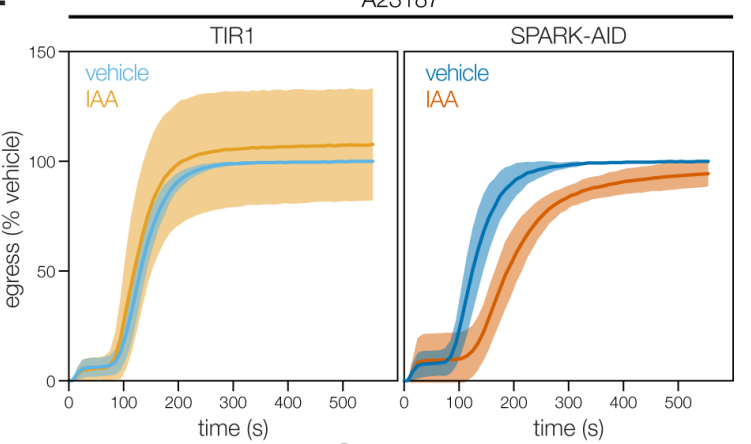

H

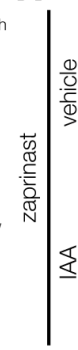
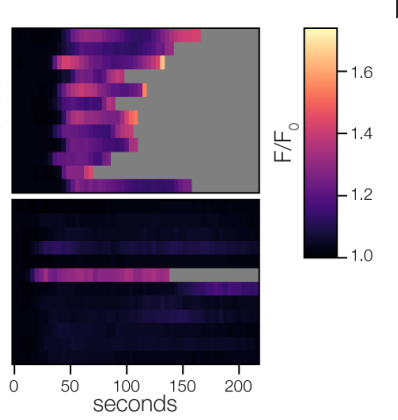

I
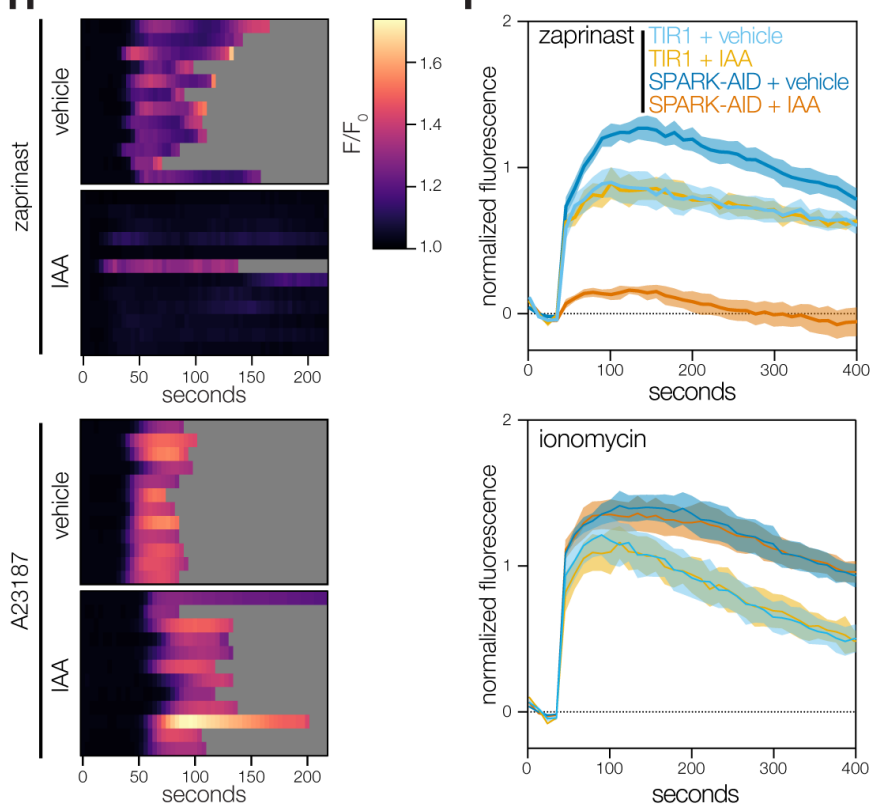

Figure 5. SPARK regulates egress and invasion through modulation of intracellular $\mathbf{C a}^{2+}$ stores. (A) Neighbor-joining phylogenetic tree of kinase domains from representative apicomplexan species, along with mammalian PDK1 orthologues and related AGC kinases. Bootstraps determined from 1000 simulations. Scale indicates substitutions per site. (B) Models of the canonical mammalian PDK1 and the apicomplexan SPARK proteins. The kinase domains, mammalian pleckstrin homology 
(PH) domain, and conserved apicomplexan MKXGFL motif are shown. (C) SPARK-AID was visualized by immunofluorescence microscopy and immunoblotting using the V5 epitope. SPARK-AID was undetectable after $24 \mathrm{~h}$ of IAA treatment. Staining for CDPK1 was used to identify parasites, and nuclei were stained with DAPI. Channels adjusted equivalently across all samples. (D) SPARK-AID depletion, as in C, monitored by immunoblot. SPARK-AID is expected to run at $98 \mathrm{kDa}$. (E-F) Parasite egress stimulated with zaprinast $(\mathrm{E})$ or the $\mathrm{Ca}^{2+}$ ionophore $\mathrm{A} 23187(\mathrm{~F})$ following $24 \mathrm{~h}$ of treatment with vehicle or IAA. Egress was monitored by the number of host cell nuclei stained with DAPI over time. Mean \pm S.D. graphed for $n=3$ biological replicates. (G) Selected frames from live video microscopy of zaprinast- or A23187-stimulated SPARK-AID parasites expressing the genetically encoded $\mathrm{Ca}^{2+}$ sensor GCaMP6f. Parasites were grown for $24 \mathrm{~h}$ with vehicle or IAA prior to the stimulation of egress. (H) Kymographs showing normalized fluorescence per vacuole relative to the initial intensity, for 12 vacuoles per strain from the experiments in G. Gray areas represent the period following egress of the vacuole under observation. (I) Extracellular parasites in basal $\mathrm{Ca}^{2+}$ buffer stimulated with zaprinast or the $\mathrm{Ca}^{2+}$ ionophore ionomycin, following $24 \mathrm{~h}$ of treatment with vehicle or IAA. Cytosolic $\mathrm{Ca}^{2+}$ flux was measured in bulk as GCaMP6f fluorescence normalized to the initial and maximum fluorescence following aerolysin permeabilization in $2 \mathrm{mM} \mathrm{Ca}^{2+}$.

As expected, SPARK was readily depleted following $24 \mathrm{~h}$ of IAA treatment in the conditional mutant, as determined by immunofluorescence microscopy and immunoblotting (Fig. 5C-5D, and S4). The protein displayed a diffuse cytosolic localization by immunofluorescence, which did not clarify its role during invasion. We carried out replication and egress assays to further characterize SPARK's role in the lytic cycle. We did not observe a decrease in replication following $24 \mathrm{~h}$ of IAA treatment, narrowing the function of SPARK to the lytic cycle stages associated with parasite motility (Fig. S5). Since egress shares several signaling pathways with invasion, we examined the role of SPARK in this process. We induced parasites to egress from host cells by increasing cGMP levels with the phosphodiesterase inhibitor zaprinast. The resulting surge in cGMP activates PKG, which triggers release of intracellular $\mathrm{Ca}^{2+}$ stores ${ }^{85-}$ ${ }^{90}$. The $\mathrm{Ca}^{2+}$ ionophore A23187 was used as an alternative trigger for egress, which, despite converging with the PKG pathway at the point of increasing cytosolic $\mathrm{Ca}^{2+}$ concentrations, has been shown to circumvent altered guanylate cyclase activity ${ }^{91-93}$. We used DAPI entry into host cells as a kinetic readout of egress ${ }^{94}$. SPARK knockdown completely blocked zaprinastinduced egress (Fig. 5E). By contrast, SPARK appeared almost entirely dispensable for A23187-induced egress, with knockdown causing only a slight delay in egress (Fig. 5F). These results suggest that SPARK loss interferes with the ability of PKG to trigger intracellular $\mathrm{Ca}^{2+}$ release.

To confirm SPARK's role in $\mathrm{Ca}^{2+}$ release, we tagged the endogenous gene via HiT vector with a mCherry-mAID epitope in parasites expressing TIR1 and the genetically-encoded fluorescent $\mathrm{Ca}^{2+}$ sensor GCaMP6f ${ }^{95}$. Treatment of GCaMP6f/SPARK-AID parasites with IAA severely decreased levels of cytosolic $\mathrm{Ca}^{2+}$ fluxes in intracellular parasites following zaprinaststimulation (Fig. 5G \& $\mathbf{5 H}$ ). As with egress, this phenotype could be rescued via A23187 treatment. Rises in parasite cytosolic $\mathrm{Ca}^{2+}$ can be caused by either release of intracellular $\mathrm{Ca}^{2+}$ stores or import of extracellular $\mathrm{Ca}^{2+}{ }^{96,97}$. To determine whether SPARK knockdown blocks intracellular $\mathrm{Ca}^{2+}$ release, we stimulated extracellular parasites in a buffer containing basal $\mathrm{Ca}^{2+}$ concentrations ( $\sim 100 \mathrm{nM}$ free $\left.\mathrm{Ca}^{2+}\right)$, removing the contribution of extracellular $\mathrm{Ca}^{2+}$ to observed changes in GCaMP fluorescence. As observed for intracellular parasites, SPARK knockdown blocked zaprinast-induced release of intracellular $\mathrm{Ca}^{2+}$ stores (Fig. 5I \& S6). By contrast, the response to the $\mathrm{Ca}^{2+}$ ionophore ionomycin was unchanged by SPARK knockdown, indicating that intracellular $\mathrm{Ca}^{2+}$ stores are intact but unresponsive to zaprinast in these parasites. These results establish SPARK as a critical regulator of the parasite intracellular $\mathrm{Ca}^{2+}$ store discharge that precedes both invasion and egress. Due to SPARK's divergence from its closest mammalian ortholog PDK1, including a lack of a phosphoinositide-binding domain, we renamed the kinase Store Potentiating/Activating Regulatory Kinase (SPARK), to more aptly describe its proposed role in apicomplexan biology. 


\section{DISCUSSION}

We present a novel platform for arrayed and pooled screening in T. gondii that provides spatiotemporal resolution to the phenotypes of target genes. Using CRISPR/Cas9-mediated repair, we targeted 155 genes for tagging with mNeonGreen and AID. This system enabled both localization and phenotypic analysis of the kinome. By utilizing pooled and arrayed screens we identified novel regulators of various processes throughout the lytic cycle. Arrayed screening assigned subcellular localizations to 39 proteins and phenotypes to 22-a majority of which were previously unknown. Pooled screening simultaneously profiled every target gene and differentiated between acute and delayed-death phenotypes. Among the genes associated with delayed death, we identified two previously uncharacterized regulators of invasion, one of which we characterize as a novel regulator of intracellular $\mathrm{Ca}^{2+}$ store release we named SPARK. We demonstrate that the HiT vector platform can be deployed at scale, with minimal automation, and is amenable to any 96-well plate-based assay. HiT vector screens provide improvements on existing technologies to increase the resolution with which we can probe apicomplexan biology.

We demonstrate that HiT screens can exploit the benefits of both arrayed and pooled formats. Arrayed screens provide information for individual clones, enabling the characterization of subcellular localizations and cellular defects, even in cases when they are polymorphic or incompletely penetrant. Pooled screening, by contrast, can compare all targeted genes simultaneously and assess more subtle fitness defects that are only apparent in competitive settings. Arrayed screening for protein localization in the kinetoplastid parasite Trypanosoma brucei successfully assigned localizations to most protein-coding genes via high-throughput transfections and microscopy of the resulting heterogeneous populations ${ }^{37}$. However, these approaches were not designed to generate clonal mutants nor to assess their phenotypes through knockdown. Pooled screens in T. brucei, Plasmodium spp., and T. gondii have examined genetic contributions to fitness in a variety of settings, but lack temporal and subcellular resolution ${ }^{12-14,98,99}$. The arrayed and pooled screening approaches of the HiT vector system work in tandem to achieve protein localization and phenotypic resolution inaccessible to existing screening platforms. Further, every well of the HiT vector array is a clonally derived strain and enables unambiguous localization of the tagged species and uniform knockdown for robust phenotypic evaluation. The arrays also enable recovery of individual clones for follow-up studies of individual genes.

While false positives in the HiT screens were minimal, 29 genes previously reported to be fitness-conferring ${ }^{12}$ did not score as such in our pooled HiT screens. These false-negatives likely originate from poor-quality gRNAs, inaccessibility of the TIR1 system to the tagged protein, and tagging-induced hypomorphism. The reduced abundance of low-quality gRNAs in the transfected population also caused skewed sampling of gRNAs in the array. Future screens should utilize more gRNAs per gene, avoid gRNAs targeting NAG PAMs, and isolate clones as early as possible for the arrays. Another subset of genes were either refractory to tagging or conditional degradation. Two kinases within the array could be localized but failed to respond to IAA treatment, possibly due to the inaccessibility of TIR1. We had foreseen this limitation and excluded predicted rhoptry kinases from our library due to their compartmentalization within secretory organelles. Since the HiT payload could constitute regulatory elements instead of a protein tag, transcriptional regulation could be used to target IAA-insensitive targets. Finally, several gRNAs dropped out of the vehicle-treated population even after correcting for 
differences between PAMs, indicating tag-induced hypomorphism. The AID system may lead to basal degradation, even in the absence of IAA, causing loss-of-function defects on target proteins and reducing the signal for microscopy ${ }^{100}$. Recently developed degron systems have minimized basal degradation and may help expand the number of proteins that are observable and tolerate the degron tag ${ }^{39,101}$. The modularity of the HiT vector system enables it to be adapted to a wide variety of biological questions.

We identified several previously unstudied regulators of the parasite lytic cycle. We classified microscopy-based phenotypes into six distinct categories representing defects across the replicative cycle. Knockdown of any of four nuclear kinases entirely blocked progression through the replicative cycle, causing an arrest at the single-parasite stage. Such kinases are likely regulators of critical checkpoints, such as the ortholog of TLK2, which promotes DNA replication in human cells ${ }^{58-60}$. By contrast, depletion of two other kinases resulted in completion of a single replicative cycle prior to an arrest as doublets. Neither gene has been studied in T. gondii; however, TGGT1_210830 is a putative ortholog of RIO kinase 1, a factor critical for ribosomal maturation and cell cycle progression ${ }^{68}$. This raises the possibility that, following TGGT1_210830 knockdown, defects in biogenesis cause ribosomes to become limiting after a round of parasite replication. Doublet phenotypes may therefore result from restriction of resources, rather than failed checkpoint as predicted for the singlet phenotypes. Other phenotypes observed by microscopy included abnormalities in parasite morphology. Multiple genes displayed defects similar to those previously observed for TgMAPK-L1 and TgMAPK2 ${ }^{63-66}$. Aberrant morphologies allude to failure in processes such as proper daughter cell assembly or cytokinesis, rather than the complete arrest in division observed as singlet and doublet phenotypes. The visualization of cellular consequences following gene knockdown expands upon general fitness screening and enables the generation of specific hypotheses regarding gene function. Further, our observed cellular defects represent acute and lethal consequences for the parasites following knockdown, establishing these kinases as promising therapeutic targets.

The temporal resolution of the HiT screen enabled the identification of genes displaying a delayed-death phenotype. We further characterized TGGT1_268210 (SPARK) and identified it as a regulator of parasite invasion and egress through the potentiation of intracellular $\mathrm{Ca}^{2+}$ stores. The proposed name for the kinase reflects the role we have defined for it in the regulation of $\mathrm{Ca}^{2+}$ stores during the parasite lytic cycle. Conditional knockdown of SPARK demonstrated that it is critical for egress following stimulation of the cGMP pathway. SPARK knockdown blocked zaprinast-induced egress and release of intracellular $\mathrm{Ca}^{2+}$ stores, phenotypes that were rescued by treatment with $\mathrm{Ca}^{2+}$ ionophores. This phenotypic pattern mirrors the regulation of the basal accumulation of filamentous actin during parasite gliding motility, defined as F-actin flux ${ }^{102}$. F-actin flux is essential for proper invasion and egress and is dependent on the $\mathrm{Ca}^{2+}$ activated kinase CDPK1 but independent of microneme protein secretion. Following treatment with the phosphodiesterase inhibitor BIPPO-which is thought to act in an analogous manner to zaprinast-F-actin flux was blocked by chemical inhibition of PKG and rescued by A23187. This indicates that regulation and potentiation of $\mathrm{Ca}^{2+}$ stores by proteins such as SPARK is a crucial event preceding F-actin mobilization, microneme protein secretion, and subsequent egress and invasion.

SPARK exhibits a phenotypic profile distinct from PKG, for which knockdown or chemical inhibition cannot be rescued by $\mathrm{Ca}^{2+}$ ionophore ${ }^{23,47,85}$. This is consistent with previously proposed models in which PKG regulates the activity of phosphoinositide phospholipase C 
(PI-PLC) leading to the production of the signaling molecules diacylglycerol (DAG) and inositol trisphosphate $\left(\mathrm{IP}_{3}\right)^{42,44,92,103-105} . \mathrm{IP}_{3}$ stimulates intracellular $\mathrm{Ca}^{2+}$ release and DAG is converted to phosphatidic acid (PA). The convergence of both the PA and $\mathrm{Ca}^{2+}$ signals is proposed to be necessary for efficient motility and secretion of microneme and rhoptry contents ${ }^{106}$. Altering the activity of the cGMP-producing guanylate cyclase (GC) by either knockdown of the guanylate cyclase itself or its accessory proteins also leads to a block in zaprinast- and BIPPOinduced egress. However, reports differ on the effect of A23187 treatment on egress following GC knockdown, ranging from a nearly normal activation of egress to a complete failure to initiate the process ${ }^{91-93}$. This inconsistency makes it difficult to definitively place SPARK in the pathway; nevertheless, we favor a model that places SPARK downstream of PKG, mediating its role releasing intracellular $\mathrm{Ca}^{2+}$ stores.

The observed phenotypes are also inconsistent with the canonical role of PDK1 activating other AGC kinases 107,108. As discussed above, failure to activate PKG would block both zaprinast- and ionophore-stimulated egress ${ }^{85}$. Analogously, failure to activate PKA, a negative regulator of egress, would result in premature egress ${ }^{45,47}$. Instead, our study indicates that SPARK functions as a positive regulator of invasion and egress via potentiation or activation of intracellular $\mathrm{Ca}^{2+}$ stores. This action could be achieved via direct stimulation or activation of $\mathrm{Ca}^{2+}$ channels, modulation of upstream positive regulators of $\mathrm{Ca}^{2+}$ release such as the $\mathrm{GC}$, or inhibition of a negative regulator of $\mathrm{Ca}^{2+}$ release such as PKA. Further work will be needed to distinguish between these models. The characterization of SPARK provides a crucial molecular handle to study the activation of intracellular $\mathrm{Ca}^{2+}$ stores-an event that mediates key transitions in the apicomplexan life cycle. The divergence of SPARK from its closest mammalian orthologs, in addition to its essential role in the life cycle, also highlights it as a potential therapeutic target.

The HiT vector screening system expands upon current screening platforms and enables the identification of complex cellular phenotypes. The HiT vector platform is scalable and offers the versatility to investigate many aspects of parasite biology. While we chose to target the kinome, this technology can handle much larger gene sets ( 2000 genes) in its current format. The AID system is also reversibile ${ }^{22}$, which could help distinguish temporary arrests in replication from lethal disruptions to identify promising drug targets. Alternative tagging epitopes, such as split GFP, can also extend HiT screening to additional biological questions. The incorporation of additional high-throughput technologies will further expand the scope and scale of the HiT vector platform. For example, high-content imaging can be combined with in situ sequencing to decode the integrated gRNA of single cells within a pooled population ${ }^{109}$. This technology would enable the assignment of phenotypes from morphological information in a pooled setting. The throughput and imaging capabilities of the HiT vector platform can also be increased with automation and improved microscopy, as have been employed for recent protein localization efforts in human cells ${ }^{36}$. Concurrently with our work, Jimenez-Ruiz and colleagues developed an alternative strategy for high-throughput phenotypic analysis of $T$. gondii, which implemented a rapamycin-inducible split Cas 9 to precisely time gene disruption. Using a parasite strain encoding indicators of actin dynamics and apicoplast segregation, they screened a library targeting 320 genes and identified two novel, essential genes required for independent steps during host cell egress and invasion. Together with the HiT screens reported herein, these technologies offer unprecedented spatiotemporal resolution to screening in T. gondii and are powerful tools for dissecting the biology of these ubiquitous apicomplexan parasites. 


\section{MATERIALS \& METHODS}

\section{Parasite and host cell culture}

T. gondii parasites were grown in human foreskin fibroblasts (HFFs) maintained in DMEM (GIBCO) supplemented with $3 \%$ inactivated fetal calf serum (IFS) and $10 \mu \mathrm{g} / \mathrm{mL}$ gentamicin (Thermo Fisher Scientific), referred to as D3. Where noted, DMEM supplemented with 10\% IFS and $10 \mu \mathrm{g} / \mathrm{mL}$ gentamicin was used, referred to as D10.

\section{Parasite transfection}

Parasites were passed through $3 \mu \mathrm{m}$ filters, pelleted at $1000 \times \mathrm{g}$ for $10 \mathrm{~min}$, washed, resuspended in Cytomix $\left(10 \mathrm{mM} \mathrm{KPO}_{4}, 120 \mathrm{mM} \mathrm{KCl}, 150 \mathrm{mM} \mathrm{CaCl}_{2}, 5 \mathrm{mM} \mathrm{MgCl}_{2}, 25 \mathrm{mM} \mathrm{HEPES}, 2 \mathrm{mM}\right.$ EDTA, $2 \mathrm{mM}$ ATP, and $5 \mathrm{mM}$ glutathione), and combined with transfected DNA to a final volume of $400 \mu \mathrm{L}$. Electroporation used an ECM 830 Square Wave electroporator (BTX) in $4 \mathrm{~mm}$ cuvettes with the following settings: $1.7 \mathrm{kV}, 2$ pulses, $176 \mu$ s pulse length, and $100 \mathrm{~ms}$ interval.

\section{Strain generation}

Oligos were ordered from IDT. All cloning was performed with Q5 $2 \times$ master mix (NEB) unless otherwise noted. Primers and plasmids used or generated in this study can be found in (Table S2).

Scarless CDPK1-mNG-AID. The V5-TEV-mNeonGreen-AID-Ty cassette was PCR amplified from plasmid BM216 and appended with repair homology arms using primers P108 and P109. Oligos P110 and P111 were duplexed and cloned into plasmid pSS013 to create the gRNA/ Cas9-expression plasmid. The resulting gRNA/Cas9-expression plasmid was co-transfected with the repair template into TIR1 parasites ${ }^{23,24}$. Following the first lysis, mNeonGreen positive clones were isolated via fluorescence-activated cell sorting. Single clones were obtained by limiting dilution and verified by PCR amplification using primers P114 and P115 and sequencing with primers $\mathrm{P} 112$ and $\mathrm{P} 113$.

Scarless CDPK3-mNG-AID. The CDPK3-mNG-AID scarless strain was generated as CDPK1mNG-AID above, using primers P116 and P117 for repair template amplification, oligos P118 and P119 for assembly of the gRNA/Cas9-expression plasmid, primers P120 and P121 for amplification of the integrated tag in clonal isolates, and primers P112 and P113 for the sequencing of tag junctions in clonal isolates.

TIR1/IMC1-tdTomato. The primers P96 and P97 were used to PCR-amplify the sequence pTUB1_IMC1-tdTomato_DHFR to yield a repair template with homology to the $5^{\prime}$ and $3^{\prime}$ ends of a defined, neutral genomic locus ${ }^{110}$. Approximately $2 \times 10^{7}$ extracellular TIR1 parasites were transfected with $50 \mu \mathrm{g}$ gRNA/Cas9 plasmid targeting the neutral genomic locus and $6 \mu \mathrm{gg}$ of repair template. Single clones were isolated by fluorescence-activated cell sorting into 96well plates containing uninfected HFFs. IMC1-tdTomato positive clones were subsequently identified by microscopy and verified by PCR amplification of the neutral genomic locus using primers P98 and P99.

TGGT1_268210, TGGT1_204280, TGGT1_239885, and TGGT1_239420 AlD-tagged lines. HiT vector cutting unit gBlocks (IDTDNA) (P122-125) were cloned via NEBuilder HiFi assembly (NEB) into the pGL015 empty mNeonGreen HiT vector backbone. HiT vectors were linearized with Bsal and co-transfected with the pSS014 Cas9-expression plasmid into TIR1 parasites. Parasite populations were selected with pyrimethamine and single clones were isolated via 
limiting dilution. Clones were verified by PCR amplification and sequencing of the junction between the $3^{\prime}$ end of the gene (primers P100-P103) and 5' end of the protein tag (primer P104 for TGGT1_268210 and primer P105 for TGGT1_204280, TGGT1_239420, and TGGT1_239885).

TIR1/GCaMP6f. The primers P106 and P107 were used to PCR-amplify the sequence pTUB1_GCaMP6f_DHFR3'UTR from plasmid Genbank MT345687 to yield a repair template with homology to the $5^{\prime}$ and $3^{\prime}$ ends of a defined, neutral genomic locus ${ }^{110}$. Approximately $1 \times 10^{7}$ extracellular parasites were transfected with $25 \mu \mathrm{g}$ gRA/Cas9-expression plasmid BM188 and $5 \mu \mathrm{g}$ GCaMP6f repair template. Following two rounds of fluorescence-activated cell sorting, GFP-positive clones were isolated by limiting dilution.

TIR1/GCaMP6f/268210-AID. The TGGT1_268210 HiT vector cutting unit gBlock (IDTDNA) was cloned into the empty V5-TEV-mCherry-AID HiT vector backbone. The HiT vector was linearized with Bsal and co-transfected with the pSS014 Cas9-expression plasmid into TIR1/ GCaMP6f parasites. Parasite populations were selected with $25 \mu \mathrm{g} / \mathrm{mL}$ mycophenolic acid and $50 \mu \mathrm{g} / \mathrm{mL}$ xanthine. Single clones were isolated via limiting dilution. Clones were verified by sequencing of the junction between the $3^{\prime}$ end of the gene and $5^{\prime}$ end of the protein tag.

\section{Analysis of CDPK1- and CDPK3-tagged HiT vector populations}

TIR1 parasites were co-transfected with $40-50 \mu \mathrm{g}$ of Bsal-linearized HiT $3^{\prime}{ }_{\text {SAG1 }}$ or HiT $3^{\prime}{ }_{\text {CDPK3 }}$ vectors and the Cas9-expression plasmid pSS014. $24 \mathrm{~h}$ post-transfection parasite populations were selected with $3 \mu \mathrm{M}$ pyrimethamine. Following pyrimethamine selection populations were analyzed by flow cytometry with a Miltenyi MACSQuant VYB. Populations were imaged by microscopy using a 60x objective and an Eclipse Ti microscope (Nikon) with an enclosure maintained at $37^{\circ} \mathrm{C}$ and $5 \% \mathrm{CO}_{2}$. For IAA-induced depletion experiments, intracellular parasites were treated with either $50 \mu \mathrm{M}$ IAA or an equivalent dilution of PBS for $24 \mathrm{~h}$. Following treatment, parasites were passed through a 27-gauge needle, isolated by filtration, and analyzed by flow cytometry with a Miltenyi MACSQuant VYB.

\section{Design and cloning of HiT vector libraries}

Three gRNA constructs were designed against each gene in the 155 gene library. The 3' end of the genomic sequences (release 36, ToxoDB.org) were scanned for gRNAs containing either NGG or NAG PAMs and cutting sites within 50 bp downstream of the stop codon. gRNAs were ranked based on predicted on-target activity and off-target activity, as determined by the Rule Set 2 and Cutting Frequency Determination calculators ${ }^{53}$, respectively, and by the distance of the cutsite from the gene's stop codon. As the Rule Set 2 calculator does not take into account efficiencies of different PAMs, the on-target scores of NAG gRNAs were penalized as predicted by the Cutting Frequency Determination calculator. These ranks were used to create an aggregate rank for gRNA selection. Initially, only the highest ranking gRNAs were selected with cutsites within $30 \mathrm{bp}$ of the stop codon and with a Rule Set 2 score above 0.2. If a gene did not have 3 gRNAs meeting these criteria, the criteria were processively relaxed to allow any gRNA within $30 \mathrm{bp}$, any gRNA within $50 \mathrm{bp}$ and with a Rule Set 2 score above 0.2, and finally any gRNA within $50 \mathrm{bp}$. A 'G' was preprended to any gRNAs that did not start with one to ensure proper RNA polymerase III initiation. Synonymous point mutations were introduced to $\mathrm{H} 1$ homology regions containing Bsal or Ascl restriction sites, in order to prevent restriction enzyme cutting during the cloning process. The guide library was synthesized by Agilent and each oligo includes a gRNA, 40 bp homology regions, an Ascl restriction site for insertion of 
the gRNA scaffold, and tandem Bsal sites for linearization of the final constructs, all flanked by sequences for cloning into empty HiT vectors (Table S2). The HiT $3^{\prime}{ }_{\text {SAG1 }}$ and HiT $3^{\prime}{ }_{\text {CDPK } 3}$ libraries were cloned as follows. The HiT $3^{\prime}{ }_{\text {CDPK3 }}$ library protocol resulted in both a slight increase of correctly assembled products and greater library diversity.

HiT 3' ${ }_{\text {SAG1 }}$ library. The synthesized oligo library was PCR amplified with primers P1 and P2. PCR products were cloned into the pTS018 entry vector via Gibson assembly (VWR). The gRNA scaffold was amplified from pSL001 with primers P3 and P4. The amplified scaffold was inserted into Ascl-digested entry vector library via NEBuilder HiFi assembly. Finally, the assembled cutting units (containing gRNA, scaffold, homology regions, and tandem Bsal sites) were PCR amplified using primers P1 and P2 and cloned into pTS020, the mNeonGreen-AID HiT $3^{\prime}$ SAG1 vector, via NEBuilder HiFi assembly. All PCR steps were performed with iProof High-Fidelity DNA polymerase (Bio-Rad) and cloning products were electroporated into MegaXDH10B $T 1^{\mathrm{R}}$ electrocompetent cells. DNA products were isolated from liquid cultures using either a ZymoPURE II Plasmid Maxiprep Kit (Zymo Research) or a Macherey-Nagel Nucleobond Xtra Maxi Kit.

HiT $3_{\text {CDPK } 3}^{\prime}$ library. In order to decrease polymerase-induced errors, during construction of the HiT $3_{\text {' }}^{\prime}{ }_{\text {DPK } 3}$ library we replaced PCR amplification steps with digestion with the type IIS restriction enzyme BsrDI. The synthesized oligo library was PCR amplified with primers P1 and P2, as in the HiT 3' ${ }_{\text {SAG1 }}$ library. PCR products were cloned into the pTS031 entry vector via NEBuilder HiFi assembly. The gRNA scaffold was isolated from pTS028 via BsrDI digestion and inserted into Ascl-digested entry vector library via NEBuilder HiFi assembly. Finally, the assembled "cutting units" were BsrDl-digested out of the entry vector library and cloned into pGL015, the mNeonGreen-AID HiT 3' ${ }_{\text {CDPK3 }}$ vector, via NEBuilder HiFi assembly. All PCR steps were performed with iProof High-Fidelity DNA polymerase and cloning products were electroporated into MegaXDH10B T1 ${ }^{\mathrm{R}}$ electrocompetent cells. DNA products were isolated from solid agar plate cultures using a ZymoPURE II Plasmid Maxiprep Kit.

\section{Pooled HiT vector screening}

For each screen, $500 \mu \mathrm{g}$ of the HiT vector library was linearized with Bsal-HFv2, cleanedup using Agencourt RNAClean XP SPRI paramagnetic beads, and co-transfected with the Cas9-expression plasmid pSS014 into $5 \times 10^{8}$ TIR1 parasites in the HiT $3^{\prime}$ SAG1 screen and $5 \times$ $10^{8}$ TIR1/IMC1-tdTomato parasites in the $3^{\prime}{ }_{\mathrm{CDPK} 3}$ screen. Parasite transfections were divided between 10 separate cuvettes. Transfected parasites were used to infect twelve and ten 15 $\mathrm{cm}^{2}$ dishes with confluent HFF monolayers in the HiT $3^{\prime}{ }_{\text {SAG1 }}$ and $3^{\prime}{ }_{\mathrm{CDPK} 3}$ screens, respectively. 3 $\mu \mathrm{M}$ pyrimethamine and $10 \mu \mathrm{g} / \mathrm{mL}$ DNasel was added $24 \mathrm{~h}$ later. The parasites were allowed to egress naturally from host cells, isolated by filtration, and passaged onto eight $15 \mathrm{~cm}^{2}$ dishes with fresh monolayers. This process was repeated for two more passages, infecting each dish with approximately $2-3 \times 10^{7}$ parasites. Following lysis of the third passage, the population was split into three $15 \mathrm{~cm}^{2}$ dishes containing fresh monolayers in D10 supplemented with 50 $\mu \mathrm{M}$ IAA and three $15 \mathrm{~cm}^{2}$ dishes in D10 supplemented with an equal dilution of vehicle (PBS). The IAA- and vehicle-treated populations were maintained and passaged in their respective conditions for three passages in the HiT $3^{\prime}{ }_{\text {AAG1 }}$ screen and for four passages in the HiT $3^{\prime}{ }_{\text {CDPK3 }}$ screen. The remaining parasites $\left(\sim 10^{8}\right)$ at select passages were pelleted and stored at $-80^{\circ} \mathrm{C}$ for analysis. Parasite DNA was extracted using the DNeasy Blood and Tissue kit (QIAGEN) and integrated gRNA constructs were amplified with primer P5 and barcoding primers P6-22. 
The resulting libraries were sequenced using a MiSeq v2 kit (Illumina) with single-reads using custom sequencing primer P23 and custom indexing primer P24.

Sequencing reads were aligned to the gRNA library. Read counts were median normalized and gRNAs in the bottom 5th percentile of the input library were removed. To account for differences in NGG and NAG PAM efficiencies, relative abundances for each gRNA were corrected for the PAM used. The PAM efficiencies were calculated by comparing the abundances of only gRNAs in the selected population that target genes identified as dispensable in a previous genome-wide knockout screen ${ }^{12}$. Fold-changes were normalized to passage 2. UMAP was used to compare gRNAs in each screen based on their pattern of fold changes for vehicle- and IAA-treated samples. Clusters were calculated by k-means. Gene centroids were calculated in UMAP space and assigned to the dispensable or fitness-conferring class using k-means clustering.

\section{Arrayed HiT vector screening}

Generation and passaging of array. In parallel to the pooled HiT $3^{\prime}{ }_{\mathrm{CDPK} 3}$ screen, single clones were isolated via limiting dilution after 4 passages of drug selection. Parasites from passage 4 were collected and sequenced as in the pooled screening experiments, using primer P5 and P25 to amplify integrated gRNA constructs. 1160 clonal isolates were arrayed into twenty 96-well plates containing HFFs. Included in each plate was a well containing the TIR1/IMC1-tdTomato parental line and a well containing the CDPK1-AID scarless strain. Arrays were passaged every 3 days with a multichannel pipette by transferring $5 \%$ of the total lysed well volume to 96 -well plates containing fresh HFF monolayers. Individual wells with incomplete lysis were scraped and passed with $10 \%$ of the total well volume.

Arrayed widefield microscopy. Freshly lysed arrays were replica plated with $12 \mu \mathrm{L}$ into 96well plates of HFFs maintained in FluoroBrite DMEM (GIBCO) supplemented with 3\% IFS, 4 mM glutamine, and $10 \mathrm{mg} / \mathrm{mL}$ gentamicin. Replica plates were centrifuged at $150 \times g$ and $18^{\circ} \mathrm{C}$ for 5 min and subsequently incubated at $37^{\circ} \mathrm{C}$ and $5 \% \mathrm{CO}_{2}$. At $3 \mathrm{~h}$ post-infection, replica plates were supplemented with either PBS or IAA to a final concentration of $50 \mu \mathrm{M}$. At $24 \mathrm{~h}$ post-IAA or PBS addition, each well was imaged at 4 adjacent fields-of-view using a 40x objective and an Eclipse Ti microscope (Nikon) with an enclosure maintained at $37^{\circ} \mathrm{C}$ and $5 \% \mathrm{CO}_{2}$.

Arrayed lytic assays. Freshly lysed arrays were replica plated with $10 \mu \mathrm{L}$ into 96-well plates of HFFs maintained in D3 supplemented with either $50 \mu \mathrm{M}$ IAA or PBS. Each replica plate contained 6 uninfected control wells. Replica plates were centrifuged at $150 \times g$ and $18^{\circ} \mathrm{C}$ for 5 min and subsequently incubated for $72 \mathrm{~h}$ at $37^{\circ} \mathrm{C}$ and $5 \% \mathrm{CO}_{2}$. Plates were washed $1 \times$ with PBS and fixed for 10 minutes with 100\% ethanol. Intact monolayers were visualized by staining the plates for 5 min with crystal violet solution (12.5 g crystal violet, $125 \mathrm{~mL} 100 \%$ ethanol, $500 \mathrm{~mL}$ $1 \%$ ammonium oxalate) followed by two PBS washes, one water wash, and overnight drying. Absorbance at $590 \mathrm{~nm}$ was read as a measure of host cell lysis. Absorbances were normalized to the average of a plate's uninfected control wells.

Dual-indexed sequencing of arrays. Parasites were harvested from $100 \mu \mathrm{L}$ of fully lysed wells by centrifugation at $1000 \times g$ and $18^{\circ} \mathrm{C}$ for $10 \mathrm{~min}$. Pellets were each resuspended in $25 \mu \mathrm{L}$ of lysis buffer (1x Q5 buffer supplemented with $0.2 \mathrm{mg} / \mathrm{mL}$ Proteinase $\mathrm{K}$ ) and lysed using the following conditions: $37^{\circ} \mathrm{C}$ for $1 \mathrm{~h}, 50^{\circ} \mathrm{C}$ for $2 \mathrm{~h}$, and $95^{\circ} \mathrm{C}$ for $15 \mathrm{~min}$. Guides were PCR-amplified from gDNA, with each well utilizing a unique combination of i7 index primers (P26-65) and i5 index primers (P66-95). PCR products from an individual plate were pooled and gel extracted 
using a Zymoclean Gel DNA Recovery Kit and subsequently pooled at equimolar ratios for sequencing. The final PCR product pool was sequenced with a MiSeq v2 kit (Illumina) using dual-indexed single-reads with primers P23 and P24. Sequencing reads were aligned to the gRNA library.

\section{Confocal microscopy of select clones}

Parasites were inoculated into 96-well glass-bottom plates (Cellvis) of HFFs maintained in FluoroBrite DMEM supplemented with 10\% IFS, $4 \mathrm{mM}$ glutamine, and $10 \mathrm{mg} / \mathrm{mL}$ gentamicin. At $24 \mathrm{~h}$ post-infection wells were imaged using an RPI spinning disk confocal microscope maintained at $37^{\circ} \mathrm{C}$ and $5 \% \mathrm{CO}_{2}$. The parental TIR1/IMC1-tdTomato strain was also imaged under identical conditions to control for background fluorescence.

\section{Plaque assays}

Parasites were inoculated into 6-well plates of HFFs maintained in D10 and either $50 \mu \mathrm{M}$ IAA or PBS and allowed to grow undisturbed for 9 days. Plates were washed with PBS and fixed for 10 min at room temperature with 100\% ethanol. Staining was performed for 5 min at room temperature with crystal violet solution, followed by two washes with PBS, one wash with water, and drying overnight.

\section{Competition assays}

Freshly lysed strains were filtered through $5 \mu \mathrm{m}$ filters and spun-down at $1000 \times g$ and $18^{\circ} \mathrm{C}$ for 10 min. Pellets were resuspended in D10 and counted. T12.5s of HFFs were infected with $1.5 \times 10^{6}$ parasites of the TIR1/IMC1-tdTomato strain and $1.5 \times 10^{6}$ parasites of the competitor strain. At $24 \mathrm{~h}$ post-infection the media of each flask was changed with fresh D10. Populations were assayed by flow cytometry following host cell lysis, and each population was passed to two wells of a 6-well plate. At $24 \mathrm{~h}$ post-infection the media of one well per strain was changed to D10 and vehicle (PBS) and the media of the second well changed to D10 and $50 \mu \mathrm{M}$ IAA. Populations were passed and maintained in these conditions every two days for four passages. Following each lysis the populations were assayed by flow cytometry with a Miltenyi MACSQuant VYB. The fraction of the population that was tdTomato negative was represented as a ratio of the [percent tdTomato negative of the IAA sample]/[percent tdTomato negative of the vehicle sample] and was normalized to the initial fraction pre-splitting into +/- IAA media.

\section{Invasion assays}

Freshly lysed strains were each passed to two flasks of HFFs containing D10 media. At 3 h post-infection one flask was supplemented with vehicle (PBS) and the second flask was supplemented with IAA to a final concentration of $50 \mu \mathrm{M}$. At $27 \mathrm{~h}$ post-infection each flask was syringe-lysed and filtered through $5 \mu \mathrm{m}$ filters. Parasites were spun down at $1000 \times g$ and $18^{\circ} \mathrm{C}$ for $10 \mathrm{~min}$. Pellets were resuspended in invasion media (HEPES-buffered DMEM without phenol red) supplemented with $1 \%$ IFS to a concentration of $1 \times 10^{6}$ parasites $/ \mathrm{mL}$. $200 \mu \mathrm{L}$ of each parasite solution was added to 3 wells of a clear-bottom 96-well plate containing HFFs. The plate was spun at $290 \times g$ and room temperature for $5 \mathrm{~min}$. Plates were incubated for $10 \mathrm{~min}$ at $37^{\circ} \mathrm{C}$ to stimulate invasion. Following incubation wells were fixed with $4 \%$ formaldehyde and extracellular parasites were stained with mouse anti-SAG1 antibody ${ }^{111}$. All parasites were stained by permeabilizing with $0.25 \%$ TritonX-100 and staining with guinea pig anti-CDPK1 (Covance, 
$\left.{ }^{112}\right)$. Cells were subsequently stained with anti-guinea pig Alexa594 antibody (Invitrogen), antimouse Alexa488 antibody (Invitrogen), and Hoechst 33258 (Santa Cruz Biotechnology) nuclear dye. Samples were imaged using a Biotek Cytation3 imaging multimode reader. The number of invaded parasites per field of view was manually counted and normalized to the number of host cells in the same area. The final invasion efficiency for each replicate was normalized to the invaded parasites per host cell nuclei of the parental TIR1 vehicle sample.

\section{Phylogenetic analysis of SPARK}

SPARK homologs were identified by BLAST search against representative apicomplexan genomes. Protein kinase domains were obtained from EupathDB based on their annotation with Interpro domain IPR011009. Sequences were curated for Theileria spp., Cryptosporidium parvum, Cryptosporidium hominis, Sarcocystis neurona, Vitrella brassicaformis, and Chromera velia to correct errors in the gene model. Domains from the nearest human, mouse, and macaque orthologues (as determined by BLAST) were used as outgroups. Individual domains were extracted from each sequence and aligned using ClustalX2, and the phylogenetic tree was generated by neighbor-joining. Visualizations were generated using FigTree (v1.4.4).

\section{Immunoblotting}

TIR1 and SPARK-AID parasites were grown in D10 for $3 \mathrm{~h}$ before being treated with either 50 $\mu \mathrm{M}$ IAA or vehicle (PBS). After $24 \mathrm{~h}$ of IAA or vehicle treatment, parasites were scraped, passed through 27-gauge needles, and isolated via filtration. Parasite pellets were resuspended in lysis buffer (0.8\% IGEPAL-CA630, $0.25 \mathrm{U} / \mu \mathrm{L}$ benzonase, and 2x Halt Protease Inhibitor Cocktail in PBS) and incubated on ice for $15 \mathrm{~min}$. Lysates were combined with 1x Laemmli buffer (diluted from 5x buffer containing 10\% SDS, 50\% glycerol, $300 \mathrm{mM}$ Tris $\mathrm{HCl} \mathrm{pH} 6.8,0.05 \%$ bromophenol blue) with $1 \%$ final volume $\beta$-mercaptoethanol and boiled for $10 \mathrm{~min}$. Samples were run on a $7.5 \%$ SDS-PAGE gel (BioRad) and transferred overnight onto a nitrocellulose membrane in transfer buffer (25 mM Tris-HCl, $192 \mathrm{mM}$ glycine, 20\% methanol). Blocking and all subsequent antibody incubations were performed in 5\% milk in TBS-T (20 mM Tris, $138 \mathrm{mM}$ $\mathrm{NaCl}, 1 \mathrm{LPBS}, 0.1 \%$ Tween-20). The blot was incubated for $1 \mathrm{~h}$ at room temperature followed by incubation with the mouse anti-V5 primary antibody (R960-25, Invitrogen) for $1 \mathrm{~h}$ rocking at room temperature. The blot was washed three times with TBS-T and incubated for $1 \mathrm{~h}$ with the anti-mouse secondary antibody (LI-COR). Following imaging the blot was incubated overnight at $4^{\circ} \mathrm{C}$ with the guinea pig anti-CDPK1 primary antibody (Covance, ${ }^{112}$ ). The blot was washed three times with TBS-T, incubated for $1 \mathrm{~h}$ at room temperature with anti-guinea pig secondary antibody (LI-COR), washed 3x with TBS-T, and imaged. Imaging was performed using a LICOR Odyssey CLx.

\section{Immunofluorescence assays}

Parasites were inoculated onto coverslips containing HFFs and after $3 \mathrm{~h}$ were treated with either $50 \mu \mathrm{M}$ IAA or PBS. At $24 \mathrm{~h}$ post-IAA addition, intracellular parasites were fixed with $4 \%$ formaldehyde and permeabilized with $0.25 \%$ Triton X-100 in PBS. Nuclei were stained with Hoechst 33342 or Hoescht 33258 (Santa Cruz) and coverslips were mounted in Prolong Diamond (Thermo Fisher). V5 was detected using a mouse monoclonal antibody (R960-25, Invitrogen). CDPK1 was detected using a guinea pig-derived polyclonal antibody (Covance) ${ }^{112}$. Primary antibodies were stained with anti-guinea pig and anti-V5 Alexa-Fluor-labeled secondary 
antibodies (Invitrogen). Images were acquired with an Eclipse Ti microscope (Nikon) using the NIS elements imaging software.

\section{Replication assays}

Parasites were inoculated onto coverslips containing HFFs and after $3 \mathrm{~h}$ were treated with either $50 \mu \mathrm{M}$ IAA or PBS. At $24 \mathrm{~h}$ post-IAA addition, intracellular parasites were fixed, permeabilized, and stained as described under "Immunofluorescence assays". For each sample, multiple fields of view were acquired with an Eclipse Ti microscope (Nikon) and the number of nuclei per vacuole were calculated from 100 vacuoles. Results are the mean of three independent experiments.

\section{Egress assays}

Egress was quantified in a plate-based manner. HFF monolayers in a clear bottomed 96-well plate infected with $7.5 \times 10^{4}$ parasites per well of parental or SPARK-AID for $3 \mathrm{~h}$ were treated with $50 \mu \mathrm{M}$ IAA or PBS for an additional $24 \mathrm{~h}$. Before imaging, the media was exchanged for FluoroBrite supplemented with 10\% IFS. Three images were taken before zaprinast (final concentration $500 \mu \mathrm{M}$ ) or A23187 (final concentration $8 \mu \mathrm{M}$ ) and DAPI (final concentration 5 $\mathrm{ng} / \mathrm{mL}$ ) were added, and imaging of DAPI-stained host cell nuclei continued for 9 additional minutes before $1 \%$ Triton X-100 was added to all wells to determine the total number of host cell nuclei. Imaging was performed at $37^{\circ} \mathrm{C}$ and $5 \% \mathrm{CO}_{2}$ using a Biotek Cytation 3 imaging multimode reader. Results are the mean of three wells per condition and are representative of three independent experiments.

\section{Live cell microscopy of GCaMP6f-expressing parasites}

To capture egress, SPARK-AID parasites were grown in HFFs in glass-bottom $35 \mathrm{~mm}$ dishes (Ibidi) for $3 \mathrm{~h}$ at which point they were treated with either $50 \mu \mathrm{M}$ IAA or PBS for an additional 24 h. Parasites were stimulated to egress with $500 \mu \mathrm{M}$ zaprinast or $8 \mu \mathrm{M}$ A23187 in Ringer's buffer prepared without $\mathrm{Ca}^{2+}\left(155 \mathrm{mM} \mathrm{NaCl}, 3 \mathrm{mM} \mathrm{KCl}, 1 \mathrm{mM} \mathrm{MgCl}, 3 \mathrm{mM} \mathrm{NaH} \mathrm{PO}_{4}, 10 \mathrm{mM} \mathrm{HEPES}\right.$, $10 \mathrm{mM}$ glucose) and supplemented with 1\% BSA (w/v) and recorded every $4 \mathrm{~s}$ for $220 \mathrm{~s}$ using an Eclipse Ti microscope (Nikon) with an enclosure maintained at $37^{\circ} \mathrm{C}$ and $5 \% \mathrm{CO}_{2}$.

\section{Extracellular ionomycin treatment of GCaMP6f strains}

Parasites were passed to $15 \mathrm{~cm}^{2}$ dishes containing HFFs and after $6 \mathrm{~h}$ treated with either 50 $\mu \mathrm{M}$ IAA or vehicle (PBS). Following $24 \mathrm{~h}$ of IAA or vehicle treatment parasites the cells were washed once with PBS. The media was changed to cold Ringer's Basal Ca ${ }^{2+}(155 \mathrm{mM} \mathrm{NaCl}, 3$ $\mathrm{mM} \mathrm{KCl}, 1 \mathrm{mM} \mathrm{MgCl}_{2}, 3 \mathrm{mM} \mathrm{NaH}_{2} \mathrm{PO}_{4}, 10 \mathrm{mM}$ HEPES, 10mM glucose, $250 \mu \mathrm{M}$ EGTA, $112 \mu \mathrm{M}$ $\mathrm{CaCl}_{2}$ ) and parasites were harvested and isolated via syringe lysis and filtration. Parasites were spun-down and washed once with Ringer's Low Ca ${ }^{2+}$ and resuspended to $1 \times 10^{7}$ parasites/ $\mathrm{mL} .100 \mu \mathrm{L}$ of parasite suspension was added to a clear-bottom 96-well plate and incubated on ice for $5 \mathrm{~min}$. Fluorescence was read with an excitation wavelength of $485 \mathrm{~nm}$ and an emission wavelength of $528 \mathrm{~nm}$ every $10 \mathrm{~s}$ in a BioTek Cytation 3. At $30 \mathrm{~s} 50 \mu \mathrm{L}$ of 3x zaprinast (100 $\mu \mathrm{M}$ final concentration), ionomycin (1 $\mu \mathrm{M}$ final concentration), or vehicle (DMSO) was added and fluorescence readings were taken for an additional $6 \mathrm{~min}$. $50 \mu \mathrm{L}$ of $4 \mathrm{x}$ aerolysin $(3 \mu \mathrm{g} / \mathrm{mL}$ final concentration) and $\mathrm{CaCl}_{2}$ (2 $\mathrm{mM}$ final concentration) was added and the assay plate was incubated at $37^{\circ} \mathrm{C}$ for $10 \mathrm{~min}$. Fluorescence was read every $1 \mathrm{~min}$ for $40 \mathrm{~min}$. The assay plate 
was shaken for $1 \mathrm{~s}$ before each read. The non-fluorescent TIR1 strain was used to perform a baseline subtraction of background fluorescence. Baseline-subtracted values were normalized to initial fluorescence pre-stimulation and maximum fluorescence post-aerolysin treatment.

\section{ACKNOWLEDGEMENTS}

We thank Bingbing Yuan for bioinformatics advice; L. David Sibley for the TIR1 strain; Wendy Salmon and the W.M Keck Blological Imaging Facility for confocal microscopy support; Peter Reddien for use of the Illumina MiSeq; Benjamin Waldman, Alice Herneisen, Elizabeth Boydston, Chris Giuliano, Alex Chan, Saima Sidik, and Benedikt Markus for technical support in generation of the array; EuPathDB and all contributors to this resource. This work was supported by funds from a National Institutes of Health grant (R01Al144369) to S.L. and a National Science Foundation Graduate Research Fellowship (2018259980) to T.A.S.

\section{REFERENCES}

1. Cabrera, D. G. et al. Plasmodial Kinase Inhibitors: License to Cure? J. Med. Chem. 61, 8061-8077 (2018).

2. Sauvey, C., Ehrenkaufer, G., Shi, D., Debnath, A. \& Abagyan, R. Antineoplastic kinase inhibitors: A new class of potent anti-amoebic compounds. PLoS Negl. Trop. Dis. 15, e0008425 (2021).

3. Merritt, C., Silva, L. E., Tanner, A. L., Stuart, K. \& Pollastri, M. P. Kinases as druggable targets in trypanosomatid protozoan parasites. Chem. Rev. 114, 11280-11304 (2014).

4. Talevich, E., Mirza, A. \& Kannan, N. Structural and evolutionary divergence of eukaryotic protein kinases in Apicomplexa. BMC Evol. Biol. 11, 321 (2011).

5. Peixoto, L. et al. Integrative genomic approaches highlight a family of parasite-specific kinases that regulate host responses. Cell Host Microbe 8, 208-218 (2010).

6. Gaji, R. Y., Sharp, A. K. \& Brown, A. M. Protein kinases in Toxoplasma gondii. Int. J. Parasitol. 51, 415-429 (2021).

7. Beraki, T. et al. Divergent kinase regulates membrane ultrastructure of the Toxoplasma parasitophorous vacuole. Proc. Natl. Acad. Sci. U. S. A. 116, 6361-6370 (2019).

8. Taylor, S. et al. A secreted serine-threonine kinase determines virulence in the eukaryotic pathogen Toxoplasma gondii. Science 314, 1776-1780 (2006).

9. Fox, B. A. et al. The Toxoplasma gondii Rhoptry Kinome Is Essential for Chronic Infection. MBio 7, (2016).

10. Fleckenstein, M. C. et al. A Toxoplasma gondii pseudokinase inhibits host IRG resistance proteins. PLoS Biol. 10, e1001358 (2012).

11. Niedelman, W. et al. The rhoptry proteins ROP18 and ROP5 mediate Toxoplasma gondii evasion of the murine, but not the human, interferon-gamma response. PLoS Pathog. 8, e1002784 (2012).

12. Sidik, S. M. et al. A Genome-wide CRISPR Screen in Toxoplasma Identifies Essential Apicomplexan Genes. Cell 166, 1423-1435.e12 (2016). 
13. Bushell, E. et al. Functional Profiling of a Plasmodium Genome Reveals an Abundance of Essential Genes. Cell 170, 260-272.e8 (2017).

14. Zhang, M. et al. Uncovering the essential genes of the human malaria parasite Plasmodium falciparum by saturation mutagenesis. Science 360, (2018).

15. Tewari, R. et al. The systematic functional analysis of Plasmodium protein kinases identifies essential regulators of mosquito transmission. Cell Host Microbe 8, 377-387 (2010).

16. Meissner, M., Brecht, S., Bujard, H. \& Soldati, D. Modulation of myosin A expression by a newly established tetracycline repressor-based inducible system in Toxoplasma gondii. Nucleic Acids Res. 29, E115 (2001).

17. Meissner, M., Schlüter, D. \& Soldati, D. Role of Toxoplasma gondii myosin A in powering parasite gliding and host cell invasion. Science 298, 837-840 (2002).

18. van Poppel, N. F. J., Welagen, J., Duisters, R. F. J. J., Vermeulen, A. N. \& Schaap, D. Tight control of transcription in Toxoplasma gondii using an alternative tet repressor. Int. J. Parasitol. 36, 443-452 (2006).

19. Pieperhoff, M. S. et al. Conditional U1 Gene Silencing in Toxoplasma gondii. PLoS One 10, e0130356 (2015).

20. Andenmatten, N. et al. Conditional genome engineering in Toxoplasma gondii uncovers alternative invasion mechanisms. Nat. Methods 10, 125-127 (2013).

21. Hunt, A. et al. Differential requirements for cyclase-associated protein (CAP) in actindependent processes of Toxoplasma gondii. Elife 8, (2019).

22. Nishimura, K., Fukagawa, T., Takisawa, H., Kakimoto, T. \& Kanemaki, M. An auxinbased degron system for the rapid depletion of proteins in nonplant cells. Nat. Methods 6 , 917-922 (2009).

23. Brown, K. M., Long, S. \& David Sibley, L. Plasma Membrane Association by N-Acylation Governs PKG Function in Toxoplasma gondii. MBio 8, (2017).

24. Long, S. et al. Calmodulin-like proteins localized to the conoid regulate motility and cell invasion by Toxoplasma gondii. PLoS Pathog. 13, e1006379 (2017).

25. Meyer, V. et al. Highly efficient gene targeting in the Aspergillus niger kusA mutant. J. Biotechnol. 128, 770-775 (2007).

26. Goins, C. L., Gerik, K. J. \& Lodge, J. K. Improvements to gene deletion in the fungal pathogen Cryptococcus neoformans: absence of Ku proteins increases homologous recombination, and co-transformation of independent DNA molecules allows rapid complementation of deletion phenotypes. Fungal Genet. Biol. 43, 531-544 (2006).

27. Kooistra, R., Hooykaas, P. J. J. \& Steensma, H. Y. Efficient gene targeting in Kluyveromyces lactis. Yeast 21, 781-792 (2004).

28. Ishibashi, K., Suzuki, K., Ando, Y., Takakura, C. \& Inoue, H. Nonhomologous chromosomal integration of foreign DNA is completely dependent on MUS-53 (human Lig4 homolog) in Neurospora. Proc. Natl. Acad. Sci. U. S. A. 103, 14871-14876 (2006). 
29. Ninomiya, Y., Suzuki, K., Ishii, C. \& Inoue, H. Highly efficient gene replacements in Neurospora strains deficient for nonhomologous end-joining. Proc. Natl. Acad. Sci. U. S. A. 101, 12248-12253 (2004).

30. Huynh, M.-H. \& Carruthers, V. B. Tagging of endogenous genes in a Toxoplasma gondii strain lacking Ku80. Eukaryot. Cell 8, 530-539 (2009).

31. Fox, B. A., Ristuccia, J. G., Gigley, J. P. \& Bzik, D. J. Efficient gene replacements in Toxoplasma gondii strains deficient for nonhomologous end joining. Eukaryot. Cel/ 8, 520529 (2009).

32. Krogan, N. J. et al. Global landscape of protein complexes in the yeast Saccharomyces cerevisiae. Nature 440, 637-643 (2006).

33. Huh, W.-K. et al. Global analysis of protein localization in budding yeast. Nature 425 , 686-691 (2003).

34. Weill, U. et al. Genome-wide SWAp-Tag yeast libraries for proteome exploration. Nat. Methods 15, 617-622 (2018).

35. Leonetti, M. D., Sekine, S., Kamiyama, D., Weissman, J. S. \& Huang, B. A scalable strategy for high-throughput GFP tagging of endogenous human proteins. Proc. Natl. Acad. Sci. U. S. A. 113, E3501-8 (2016).

36. Cho, N. H. et al. OpenCell: proteome-scale endogenous tagging enables the cartography of human cellular organization. bioRxiv 2021.03.29.437450 (2021) doi:10.1101/2021.03.29.437450.

37. Dean, S., Sunter, J. D. \& Wheeler, R. J. TrypTag.org: A Trypanosome Genome-wide Protein Localisation Resource. Trends Parasitol. 33, 80-82 (2017).

38. Sidik, S. M., Hackett, C. G., Tran, F., Westwood, N. J. \& Lourido, S. Efficient genome engineering of Toxoplasma gondii using CRISPR/Cas9. PLoS One 9, e100450 (2014).

39. Sathyan, K. M. et al. An improved auxin-inducible degron system preserves native protein levels and enables rapid and specific protein depletion. Genes Dev. 33, 1441-1455 (2019).

40. Long, S., Anthony, B., Drewry, L. L. \& Sibley, L. D. A conserved ankyrin repeatcontaining protein regulates conoid stability, motility and cell invasion in Toxoplasma gondii. Nat. Commun. 8, 2236 (2017).

41. Lourido, S. et al. Calcium-dependent protein kinase 1 is an essential regulator of exocytosis in Toxoplasma. Nature 465, 359-362 (2010).

42. Lourido, S., Tang, K. \& Sibley, L. D. Distinct signalling pathways control Toxoplasma egress and host-cell invasion. EMBO J. 31, 4524-4534 (2012).

43. McCoy, J. M., Whitehead, L., van Dooren, G. G. \& Tonkin, C. J. TgCDPK3 Regulates Calcium-Dependent Egress of Toxoplasma gondii from Host Cells. PLoS Pathog. 8, e1003066 (2012).

44. Bullen, H. E. et al. Phosphatidic Acid-Mediated Signaling Regulates Microneme Secretion in Toxoplasma. Cell Host Microbe 19, 349-360 (2016).

45. Uboldi, A. D. et al. Protein kinase A negatively regulates $\mathrm{Ca}+$ + signalling in Toxoplasma gondii. PLoS Biol. 16, e2005642 (2018). 
46. Farrell, A. et al. A DOC2 protein identified by mutational profiling is essential for apicomplexan parasite exocytosis. Science 335, 218-221 (2012).

47. Jia, Y. et al. Crosstalk between PKA and PKG controls $\mathrm{pH}$-dependent host cell egress of Toxoplasma gondii. EMBO J. 36, 3250-3267 (2017).

48. Brown, K. M., Long, S. \& Sibley, L. D. Conditional Knockdown of Proteins Using Auxininducible Degron (AID) Fusions in Toxoplasma gondii. Bio Protoc 8, (2018).

49. Paquet, D. et al. Efficient introduction of specific homozygous and heterozygous mutations using CRISPR/Cas9. Nature 533, 125-129 (2016).

50. Dewari, P. S. et al. An efficient and scalable pipeline for epitope tagging in mammalian stem cells using Cas9 ribonucleoprotein. Elife 7, (2018).

51. Bialk, P., Rivera-Torres, N., Strouse, B. \& Kmiec, E. B. Regulation of Gene Editing Activity Directed by Single-Stranded Oligonucleotides and CRISPR/Cas9 Systems. PLoS One 10, e0129308 (2015).

52. Liang, X., Potter, J., Kumar, S., Ravinder, N. \& Chesnut, J. D. Enhanced CRISPR/ Cas9-mediated precise genome editing by improved design and delivery of gRNA, Cas9 nuclease, and donor DNA. J. Biotechnol. 241, 136-146 (2017).

53. Doench, J. G. et al. Optimized sgRNA design to maximize activity and minimize offtarget effects of CRISPR-Cas9. Nat. Biotechnol. 34, 184-191 (2016).

54. Harding, C. R. et al. Gliding Associated Proteins Play Essential Roles during the Formation of the Inner Membrane Complex of Toxoplasma gondii. PLoS Pathog. 12, e1005403 (2016).

55. Barylyuk, K. et al. A Comprehensive Subcellular Atlas of the Toxoplasma Proteome via hyperLOPIT Provides Spatial Context for Protein Functions. Cell Host Microbe 28, 752-766. e9 (2020).

56. Alvarez, C. A. \& Suvorova, E. S. Checkpoints of apicomplexan cell division identified in Toxoplasma gondii. PLoS Pathog. 13, e1006483 (2017).

57. Varberg, J. M., Coppens, I., Arrizabalaga, G. \& Gaji, R. Y. TgTKL1 Is a Unique PlantLike Nuclear Kinase That Plays an Essential Role in Acute Toxoplasmosis. MBio 9, (2018).

58. Silljé, H. H., Takahashi, K., Tanaka, K., Van Houwe, G. \& Nigg, E. A. Mammalian homologues of the plant Tousled gene code for cell-cycle-regulated kinases with maximal activities linked to ongoing DNA replication. EMBO J. 18, 5691-5702 (1999).

59. Pilyugin, M., Demmers, J., Verrijzer, C. P., Karch, F. \& Moshkin, Y. M. Phosphorylationmediated control of histone chaperone ASF1 levels by Tousled-like kinases. PLoS One 4, e8328 (2009).

60. Silljé, H. H. \& Nigg, E. A. Identification of human Asf1 chromatin assembly factors as substrates of Tousled-like kinases. Curr. Biol. 11, 1068-1073 (2001).

61. Eckert, D. et al. Prp4 Kinase Grants the License to Splice: Control of Weak Splice Sites during Spliceosome Activation. PLoS Genet. 12, e1005768 (2016).

62. Naumov, A. et al. The Toxoplasma Centrocone Houses Cell Cycle Regulatory Factors. MBio 8, (2017). 
63. Gubbels, M.-J. et al. Forward genetic analysis of the apicomplexan cell division cycle in Toxoplasma gondii. PLoS Pathog. 4, e36 (2008).

64. Chen, C.-T. \& Gubbels, M.-J. The Toxoplasma gondii centrosome is the platform for internal daughter budding as revealed by a Nek1 kinase mutant. J. Cell Sci. 126, 3344-3355 (2013).

65. Suvorova, E. S., Francia, M., Striepen, B. \& White, M. W. A novel bipartite centrosome coordinates the apicomplexan cell cycle. PLoS Biol. 13, e1002093 (2015).

66. Hu, X., O'Shaughnessy, W. J., Beraki, T. G. \& Reese, M. L. Loss of the Conserved Alveolate Kinase MAPK2 Decouples Toxoplasma Cell Growth from Cell Division. MBio 11, (2020).

67. Martín Moyano, P., Němec, V. \& Paruch, K. Cdc-Like Kinases (CLKs): Biology, Chemical Probes, and Therapeutic Potential. Int. J. Mol. Sci. 21, (2020).

68. Berto, G., Ferreira-Cerca, S. \& De Wulf, P. The Rio1 protein kinases/ATPases: conserved regulators of growth, division, and genomic stability. Curr. Genet. 65, 457-466 (2019).

69. Mallari, J. P., Oksman, A., Vaupel, B. \& Goldberg, D. E. Kinase-associated endopeptidase 1 (Kae1) participates in an atypical ribosome-associated complex in the apicoplast of Plasmodium falciparum. J. Biol. Chem. 289, 30025-30039 (2014).

70. Back, P. S. et al. Ancient MAPK ERK7 is regulated by an unusual inhibitory scaffold required for Toxoplasma apical complex biogenesis. Proc. Natl. Acad. Sci. U. S. A. 117, 12164-12173 (2020).

71. O'Shaughnessy, W. J., Hu, X., Beraki, T., McDougal, M. \& Reese, M. L. Loss of a conserved MAPK causes catastrophic failure in assembly of a specialized cilium-like structure in Toxoplasma gondii. MBoC 31, 881-888 (2020).

72. Sampels, V. et al. Conditional mutagenesis of a novel choline kinase demonstrates plasticity of phosphatidylcholine biogenesis and gene expression in Toxoplasma gondii. J. Biol. Chem. 287, 16289-16299 (2012).

73. Mclnnes, L., Healy, J. \& Melville, J. UMAP: Uniform Manifold Approximation and Projection for Dimension Reduction. arXiv 1-51 (2018).

74. Hartigan, J. A. \& Wong, M. A. Algorithm AS 136: A K-means clustering algorithm. J. R. Stat. Soc. Ser. C Appl. Stat. 28, 100 (1979).

75. Katso, R. et al. Cellular function of phosphoinositide 3-kinases: implications for development, homeostasis, and cancer. Annu. Rev. Cell Dev. Biol. 17, 615-675 (2001).

76. Balla, A. \& Balla, T. Phosphatidylinositol 4-kinases: old enzymes with emerging functions. Trends Cell Biol. 16, 351-361 (2006).

77. Dvorin, J. D. et al. A plant-like kinase in Plasmodium falciparum regulates parasite egress from erythrocytes. Science 328, 910-912 (2010).

78. Kato, N. et al. Gene expression signatures and small-molecule compounds link a protein kinase to Plasmodium falciparum motility. Nat. Chem. Biol. 4, 347-356 (2008). 
79. Bansal, A. et al. Characterization of Plasmodium falciparum calcium-dependent protein kinase 1 (PfCDPK1) and its role in microneme secretion during erythrocyte invasion. J. Biol. Chem. 288, 1590-1602 (2013).

80. Green, J. L. et al. The motor complex of Plasmodium falciparum: phosphorylation by a calcium-dependent protein kinase. J. Biol. Chem. 283, 30980-30989 (2008).

81. Chen, F., Mackey, A. J., Stoeckert, C. J., Jr \& Roos, D. S. OrthoMCL-DB: querying a comprehensive multi-species collection of ortholog groups. Nucleic Acids Res. 34, D363-8 (2006).

82. Rosenberg, A., Luth, M. R., Winzeler, E. A., Behnke, M. \& Sibley, L. D. Evolution of resistance in vitro reveals mechanisms of artemisinin activity in Toxoplasma gondii. Proc. Natl. Acad. Sci. U. S. A. (2019) doi:10.1073/pnas. 1914732116.

83. Hitz, E. et al. The 3-phosphoinositide-dependent protein kinase 1 is an essential upstream activator of protein kinase $A$ in malaria parasites. bioRxiv 2021.05.27.445967 (2021) doi:10.1101/2021.05.27.445967.

84. Dittrich, A. C. N. \& Devarenne, T. P. Perspectives in PDK1 evolution: insights from photosynthetic and non-photosynthetic organisms. Plant Signal. Behav. 7, 642-649 (2012).

85. Sidik, S. M. et al. Using a Genetically Encoded Sensor to Identify Inhibitors of Toxoplasma gondii Ca2+ Signaling. J. Biol. Chem. 291, 9566-9580 (2016).

86. Howard, B. L. et al. Identification of potent phosphodiesterase inhibitors that demonstrate cyclic nucleotide-dependent functions in apicomplexan parasites. ACS Chem. Biol. 10, 1145-1154 (2015).

87. Wiersma, H. I. et al. A role for coccidian cGMP-dependent protein kinase in motility and invasion. Int. J. Parasitol. 34, 369-380 (2004).

88. Brown, K. M., Lourido, S. \& Sibley, L. D. Serum Albumin Stimulates Protein Kinase G-dependent Microneme Secretion in Toxoplasma gondii. J. Biol. Chem. 291, 9554-9565 (2016).

89. McRobert, L. et al. Gametogenesis in malaria parasites is mediated by the cGMPdependent protein kinase. PLoS Biol. 6, e139 (2008).

90. Collins, C. R. et al. Malaria parasite cGMP-dependent protein kinase regulates blood stage merozoite secretory organelle discharge and egress. PLoS Pathog. 9, e1003344 (2013).

91. Yang, L. et al. An apically located hybrid guanylate cyclase-ATPase is critical for the initiation of Ca2+ signaling and motility in Toxoplasma gondii. J. Biol. Chem. 294, 89598972 (2019).

92. Bisio, H., Lunghi, M., Brochet, M. \& Soldati-Favre, D. Phosphatidic acid governs natural egress in Toxoplasma gondii via a guanylate cyclase receptor platform. Nat Microbio/ 4 , 420-428 (2019).

93. Brown, K. M. \& Sibley, L. D. Essential cGMP Signaling in Toxoplasma Is Initiated by a Hybrid P-Type ATPase-Guanylate Cyclase. Cell Host Microbe 24, 804-816.e6 (2018).

94. Shortt, E. \& Lourido, S. Plate-Based Quantification of Stimulated Toxoplasma Egress. Methods Mol. Biol. 2071, 171-186 (2020). 
95. Chen, T.-W. et al. Ultrasensitive fluorescent proteins for imaging neuronal activity. Nature 499, 295-300 (2013).

96. Pace, D. A., McKnight, C. A., Liu, J., Jimenez, V. \& Moreno, S. N. J. Calcium entry in Toxoplasma gondii and its enhancing effect of invasion-linked traits. J. Biol. Chem. 289, 19637-19647 (2014).

97. Arrizabalaga, G. \& Boothroyd, J. C. Role of calcium during Toxoplasma gondii invasion and egress. Int. J. Parasitol. 34, 361-368 (2004).

98. Alsford, S. et al. High-throughput phenotyping using parallel sequencing of RNA interference targets in the African trypanosome. Genome Res. 21, 915-924 (2011).

99. Young, J. et al. A CRISPR platform for targeted in vivo screens identifies Toxoplasma gondii virulence factors in mice. Nat. Commun. 10, 3963 (2019).

100. Natsume, T., Kiyomitsu, T., Saga, Y. \& Kanemaki, M. T. Rapid Protein Depletion in Human Cells by Auxin-Inducible Degron Tagging with Short Homology Donors. Cell Rep. 15, 210-218 (2016).

101. Yesbolatova, A. et al. The auxin-inducible degron 2 technology provides sharp degradation control in yeast, mammalian cells, and mice. Nat. Commun. 11, 5701 (2020).

102. Tosetti, N., Dos Santos Pacheco, N., Soldati-Favre, D. \& Jacot, D. Three F-actin assembly centers regulate organelle inheritance, cell-cell communication and motility in Toxoplasma gondii. Elife 8, (2019).

103. Brochet, M. et al. Phosphoinositide metabolism links cGMP-dependent protein kinase $\mathrm{G}$ to essential $\mathrm{Ca}^{2+}$ signals at key decision points in the life cycle of malaria parasites. PLoS Biol. 12, e1001806 (2014).

104. Carruthers, V. B., Moreno, S. N. \& Sibley, L. D. Ethanol and acetaldehyde elevate intracellular [Ca2+] and stimulate microneme discharge in Toxoplasma gondii. Biochem. J 342 ( Pt 2), 379-386 (1999).

105. Lovett, J. L., Marchesini, N., Moreno, S. N. J. \& Sibley, L. D. Toxoplasma gondii microneme secretion involves intracellular $\mathrm{Ca}(2+)$ release from inositol 1,4,5-triphosphate (IP(3))/ryanodine-sensitive stores. J. Biol. Chem. 277, 25870-25876 (2002).

106. Bullen, H. E., Bisio, H. \& Soldati-Favre, D. The triumvirate of signaling molecules controlling Toxoplasma microneme exocytosis: Cyclic GMP, calcium, and phosphatidic acid. PLoS Pathog. 15, e1007670 (2019).

107. Leroux, A. E., Schulze, J. O. \& Biondi, R. M. AGC kinases, mechanisms of regulation and innovative drug development. Semin. Cancer Biol. 48, 1-17 (2018).

108. Mora, A., Komander, D., van Aalten, D. M. F. \& Alessi, D. R. PDK1, the master regulator of AGC kinase signal transduction. Semin. Cell Dev. Biol. 15, 161-170 (2004).

109. Feldman, D. et al. Optical Pooled Screens in Human Cells. Cell 179, 787-799.e17 (2019).

110. Markus, B. M., Bell, G. W., Lorenzi, H. A. \& Lourido, S. Optimizing Systems for Cas9 Expression in Toxoplasma gondii. mSphere 4, (2019). 
bioRxiv preprint doi: https://doi.org/10.1101/2021.09.23.461611; this version posted September 24,2021 . The copyright holder for this preprint (which was not certified by peer review) is the author/funder, who has granted bioRxiv a license to display the preprint in perpetuity. It is made available under aCC-BY-NC-ND 4.0 International license.

111. Burg, J. L., Perelman, D., Kasper, L. H., Ware, P. L. \& Boothroyd, J. C. Molecular analysis of the gene encoding the major surface antigen of Toxoplasma gondii. J. Immunol. 141, 3584-3591 (1988).

112. Waldman, B. S. et al. Identification of a Master Regulator of Differentiation in Toxoplasma. Cell 180, 359-372.e16 (2020). 